\title{
QUASI-DOUBLING OF SELF-SIMILAR MEASURES WITH OVERLAPS
}

\author{
KATHRYN E. HARE, KEVIN G. HARE, AND SASCHA TROSCHEIT
}

\begin{abstract}
The Assouad and quasi-Assouad dimensions of a metric space provide information about the extreme local geometric nature of the set. The Assouad dimension of a set has a measure theoretic analogue, which is also known as the upper regularity dimension. One reason for the interest in this notion is that a measure has finite Assouad dimension if and only if it is doubling.

Motivated by recent progress on both the Assouad dimension of measures that satisfy a strong separation condition and the quasi-Assouad dimension of metric spaces, we introduce the notion of the quasi-Assouad dimension of a measure. As with sets, the quasiAssouad dimension of a measure is dominated by its Assouad dimension. It dominates both the quasi-Assouad dimension of its support and the supremal local dimension of the measure, with strict inequalities possible in all cases.

Our main focus is on self-similar measures in $\mathbb{R}$ whose support is an interval and which may have 'overlaps'. For measures that satisfy a weaker condition than the weak separation condition we prove that finite quasi-Assouad dimension is equivalent to quasidoubling of the measure, a strictly less restrictive property than doubling. Further, we exhibit a large class of such measures for which the quasi-Assouad dimension coincides with the maximum of the local dimension at the endpoints of the support. This class includes all regular, equicontractive self-similar measures satisfying the weak separation condition, such as convolutions of uniform Cantor measures with integer ratio of dissection. Other properties of this dimension are also established and many examples are given.
\end{abstract}

\section{INTRODUCTION}

The Assouad dimension of a metric space is an indication of its 'thickness' and is of great use in solving embedding problems, see [1]. Recently, the Assouad dimension has attracted significant attention in the metric geometry community, especially when studying dynamical objects such as attractors and fractals, see for instance [5, 16, 19]. It gives quantitative information about the 'worst' possible scaling of a set. The quasi-Assouad dimension was introduced by Lü and Xi [17] and differs from the Assouad dimension by ignoring some subexponential effects. It is a lower bound on the Assouad dimension and an upper bound on the Hausdorff and upper box dimensions of the set. Although these dimensions will often coincide, such as for self-similar sets satisfying the open set condition, there are important examples where the dimensions are different. We refer the reader to 8 ] and 9] for deterministic and stochastic examples.

2010 Mathematics Subject Classification. 28C15; 28A80, 37C45.

Key words and phrases. self-similar measures, quasi-doubling, quasi-Assouad dimension, weak separation condition.

KEH was supported by NSERC Grant 2016-03719. KGH was supported by NSERC Grant 2014-03154. ST was supported by NSERC Grants 2014-03154 and 2016-03719, and the University of Waterloo. 
As with the Hausdorff dimension, there is an analogue of the Assouad dimension of the measure. This dimension is also known as the upper regularity dimension and was first studied by Käenmäki in [13, 14. As with the Assouad dimension of a set, it captures the worst scaling behaviour of a measure. One reason the Assouad dimension of a measure $\mu$ is of interest is because it is finite if and only if the measure is doubling [7, meaning, there is some constant $c$ such that $\mu(B(x, r)) \geq c \mu(B(x, 2 r))$ for every $x \in \operatorname{supp} \mu$.

In this article we introduce and investigate the measure-theoretic analogue of the quasiAssouad dimension. We begin the paper by defining the quasi-Assouad dimension of a measure and proving basic properties. In particular, we show that the quasi-Assouad dimension is always dominated by the Assouad dimension of the measure and always dominates all local dimensions of the measure, with strict inequalities possible in each case.

Following this introductory material, we focus mainly on the quasi-Assouad dimension of self-similar measures. As remarked in [7, "self-similar measures not satisfying the strong separation condition are typically not doubling", and thus have infinite Assouad dimension. In contrast, we show that doubling is not a requirement for finite quasi-Assouad dimension, thus it is of interest to study the quasi-Assouad dimension of self-similar measures with 'overlap', those which fail the open set condition. Our paper is primarily concerned with self-similar measures that have support equal to $[0,1]$ and arise from an iterated function system that satisfies a natural separation condition, such as the weak separation condition. One class of examples are the Bernoulli convolutions with contraction factor the inverse of a Pisot number.

There are two major objectives to this paper. The first is to characterize finite quasiAssouad dimension for such measures in terms of a geometric doubling-like condition that is strictly weaker than doubling. In particular, as a special case of Theorem 4.4, (see in particular Corollary 4.6) we obtain

Theorem A. If $\mu$ is a self-similar measure that satisfies the weak separation condition and has support $[0,1]$, then the quasi-Assouad dimension of $\mu$ is finite if and only if for every $\varepsilon>0$ there is a constant $C_{\varepsilon}$ such that

$$
\mu(B(x, r)) \geq C_{\varepsilon} r^{\varepsilon} \mu(B(x, 2 r)) \text { for all } 0<r<1 \text { and } x \in \operatorname{supp} \mu .
$$

It is easy to see that any measure that is doubling satisfies property (1.1). More generally, we also prove that any measure (whether self-similar or not) that has finite quasi-Assouad dimension satisfies this property.

Fraser and Howroyd prove in [7] that if $\mu$ is a self-similar measure that satisfies the strong separation condition, then the Assouad dimension of $\mu$ is equal to the supremal local dimension of $\mu$. Hence the quasi-Assouad dimension of the measure is also equal to its supremal local dimension. It is natural to ask if this is more generally true for self-similar measures. In fact, it need not be true even for self-similar measures that satisfy the open set condition since their quasi-Assouad dimension can be infinite; see Example 3.2 .

The second major goal of the paper is to prove that the quasi-Assouad dimension is equal to the maximum local dimension for an interesting class of 'overlapping' self-similar measures which we now briefly describe. An equicontractive self-similar measure is said to be regular if the probabilities associated with the left and right-most contractions from the underlying iterated function system are equal and minimal. Much studied examples of equicontractive, regular self-similar measures that satisfy the weak separation condition include Bernoulli convolutions with contraction ratios the inverse of Pisot numbers and 
convolutions of uniform Cantor measures on Cantor sets with integer ratios of dissection; c.f. 3, 10, 20, 22. An extension of the notion of regular to self-similar measures that are not necessarily equicontractive is known as generalized regular; see Definition [5.4, It is a consequence of Theorem 5.8 (see in particular Corollary 5.9) that

Theorem B. If $\mu$ is a generalized regular, self-similar measure that satisfies the weak separation condition and has support $[0,1]$, then the quasi-Assouad dimension of $\mu$ is

$$
\operatorname{dim}_{q A} \mu=\max \left\{\overline{\operatorname{dim}}_{\text {loc }} \mu(x): x \in \operatorname{supp} \mu\right\} .
$$

In particular, this is true for equicontractive, regular self-similar measures.

As we observe in Remark [5.13, it is not necessary for a measure to be generalized regular for its quasi-Assouad dimension to coincide with its maximum upper local dimension. This is a consequence of the bounds we obtain in Theorem 5.12 for the quasi-Assouad dimension of a larger class of sets known as weakly comparable. It is unknown if all the weakly comparable measures have the property that their quasi-Assouad dimensions coincide with their maximum upper local dimensions.

The results stated above actually hold for a strictly larger class of self-similar measures than those satisfying the weak separation condition. We call this weaker property the asymptotic gap weak separation condition. This property, described in Definition 3.5, is closely related to the asymptotic weak separation condition introduced by Feng in 4 (and may coincide with it). One reason for the interest in this property is that it is satisfied by Bernoulli convolutions with contraction ratios the inverse of Salem numbers since these measures do not satisfy the weak separation condition.

The paper is organized as follows. In Section 2 we define the quasi-Assouad dimension of a measure and prove basic properties such as the relationship with the Assouad dimension and the local dimensions of the measure. In Section 3, we introduce the asymptotic gap weak separation condition and see that it lies between the weak separation condition and the asymptotic weak separation condition. Many of our results on the quasi-Assouad dimension of self-similar measures that satisfy this separation property rely upon good estimates of the measure of net intervals, certain subintervals of $[0,1]$ that arise naturally through the iterative process. Useful technical results relating the measures of net intervals and the measures of balls can be found in Section 3. These were motivated by the study of measures of finite type; see [2, [10, or [12] for further background information on net intervals, measures of finite type, and other related notions.

In Section 4 we characterize finite quasi-Assouad dimension for measures satisfying the asymptotic gap weak separation condition in terms of the quasi-doubling property and show that all measures with finite quasi-Assouad dimension have this property. In Sections 5.1 and 5.2 we introduce weakly comparable and generalized regular measures. Our proof that the generalized regular, self-similar measures satisfying the asymptotic gap weak separation condition have quasi-Assouad dimension equal to their maximum upper local dimension is given in Section 5.3. Dimensional properties of weakly comparable measures are found in Section 5.4. We conclude the paper by giving an example that demonstrates the importance of the assumption that the support of the self-similar measure is $[0,1]$. 


\section{BASIC PROPERTIES OF THE QUASI-ASSOUAD DIMENSION}

Given $X$, a compact subset of $\mathbb{R}^{d}$, we write $N_{r}(E)$ for the least number of sets of diameter at most $r$ that are required to cover $E$. Let

$h(\delta)=\inf \left\{\alpha:\left(\exists C_{1}, C_{2}>0\right)\left(\forall 0<r<R^{1+\delta}<R<C_{1}\right) \sup _{x \in X} N_{r}(B(x, R) \cap E) \leq C_{2}\left(\frac{R}{r}\right)^{\alpha}\right\}$.

The Assouad dimension of $E$ is given by

$$
\operatorname{dim}_{\mathrm{A}} E=h(0) .
$$

The quasi-Assouad dimension is characterized by an exponential gap between $r$ and $R$ and is given by

$$
\operatorname{dim}_{\mathrm{qA}} E=\lim _{\delta \rightarrow 0} h(\delta) .
$$

Note that it is not necessary to have both constants, $C_{1}$ and $C_{2}$, in the definition above. However, we introduced both constants as it is convenient to be able to change between the two definitions.

Our interest in this paper is to study a natural analogue of the Assouad and quasi-Assouad dimension for measures. By a measure we will always mean a Borel probability measure on $\mathbb{R}^{d}$.

Definition 2.1. Given a measure $\mu$ and $\delta \geq 0$, set

$H(\delta)=\inf \left\{s:\left(\exists C_{1}, C_{2}>0\right)\left(\forall 0<r \leq R^{1+\delta} \leq R \leq C_{1}\right) \sup _{x \in \operatorname{supp} \mu} \frac{\mu(B(x, R))}{\mu(B(x, r))} \leq C_{2}\left(\frac{R}{r}\right)^{s}\right\}$.

The Assouad dimension of $\mu$ is $\operatorname{dim}_{\mathrm{A}} \mu=H(0)$ and the quasi-Assouad dimension of $\mu$ is $\operatorname{dim}_{\mathrm{qA}} \mu=\lim _{\delta \rightarrow 0} H(\delta)$.

We note that the limit must exist by monotonicity, but may be infinite. The Assouad dimension of a measure has also been referred to as the upper regularity dimension, see [7] and [14.

Since $H(\delta)$ is a non-decreasing function, we clearly have $\operatorname{dim}_{\mathrm{qA}} \mu \leq \operatorname{dim}_{\mathrm{A}} \mu$. As with the quasi-Assouad/Assouad dimensions of sets, the quasi-Assouad and Assouad dimensions of a measure need not coincide and it is even possible for the Assouad dimension of a measure to be infinite, while the quasi-Assouad dimension is finite. See Example 2.3.

In [7. Theorem 2.1] it is shown that $\operatorname{dim}_{\mathrm{A}} \operatorname{supp} \mu \leq \operatorname{dim}_{\mathrm{A}} \mu$. The analogous statement holds for the quasi-Assouad dimension of measures.

Proposition 2.2. Let $\mu$ be a Borel probability measure on $\mathbb{R}^{d}$ with bounded support. Then

$$
\begin{aligned}
\operatorname{dim}_{\mathrm{qA}} \operatorname{supp} \mu & \leq \operatorname{dim}_{\mathrm{A}} \operatorname{supp} \mu \leqslant \operatorname{dim}_{\mathrm{A}} \mu . \\
& \leqslant \operatorname{dim}_{\mathrm{qA}} \mu \leq
\end{aligned}
$$

Proof. Suppose that $s=\operatorname{dim}_{\mathrm{qA}} \mu$ and $t=\operatorname{dim}_{\mathrm{qA}} \operatorname{supp} \mu$. Then, given $\varepsilon>0$ and $\delta>0$ there exists a constant $c_{1}>0$ such that for all $r \leq R^{1+\delta}$ and all $x \in \operatorname{supp} \mu$,

$$
\frac{\mu(B(x, R))}{\mu(B(x, r))} \leq c_{1}\left(\frac{R}{r}\right)^{s+\varepsilon}
$$


Similarly, there exists $c_{2}>0$ and points $y \in \operatorname{supp} \mu$ and $r, R$ with $2 r \leq R^{1+\delta}$, such that

$$
N_{2 r}(B(y, R) \cap \operatorname{supp} \mu) \geq c_{2}\left(\frac{R}{2 r}\right)^{t-\varepsilon} .
$$

Let $B_{j}\left(x_{j}, r\right), j=1, \ldots, k$ be a maximal collection of disjoint balls with centres in $B(y, R) \cap$ $\operatorname{supp} \mu$. Then $\bigcup_{j=1}^{k} B\left(x_{j}, 2 r\right) \operatorname{covers} B(y, R) \cap \operatorname{supp} \mu$, and so $k \geq N_{2 r}(B(y, R) \cap \operatorname{supp} \mu)$.

These comments imply

$$
\mu(B(y, 2 R)) \geq k \min _{j} \mu\left(B\left(x_{j}, r\right)\right)=k \mu\left(B\left(x_{j_{0}}, r\right)\right)
$$

for a suitable choice of index $j_{0}$. Moreover, $B(y, 2 R) \subseteq B\left(x_{j_{0}}, 4 R\right)$, thus

$$
c_{2}\left(\frac{R}{2 r}\right)^{t-\varepsilon} \leq N_{2 r}(B(y, R) \cap \operatorname{supp} \mu) \leq k \leq \frac{\mu(B(y, 2 R))}{\mu\left(B\left(x_{j_{0}}, r\right)\right)} \leq \frac{\mu\left(B\left(x_{j_{0}}, 4 R\right)\right)}{\mu\left(B\left(x_{j_{0}}, r\right)\right)} \leq c_{1}\left(\frac{2 R}{r}\right)^{s+\varepsilon} .
$$

Since we can find arbitrarily small $R$ satisfying this inequality, we must have $s \geq t$.

Example 2.3. (i) A measure $\mu$ satisfying $\operatorname{dim}_{\mathrm{qA}} \operatorname{supp} \mu<\operatorname{dim}_{\mathrm{qA}} \mu<\operatorname{dim}_{\mathrm{A}} \mu$ : Let $C$ be the classic middle third Cantor set. We will label the Cantor intervals at step $n$ of the standard construction as $I_{\omega}$ where $\omega \in\{0,1\}^{n}$, with the meaning that if $I_{\nu}$ is a Cantor interval of step $n-1$, then its left descendent is the interval labelled $I_{\nu 0}$ and the right descendent is labelled $I_{\nu 1}$. Choose a sparse sequence $\left(n_{k}\right)$. Put $p_{0}^{(n)}=1 / 3, p_{1}^{(n)}=2 / 3$ if $n \neq n_{k}$ and $p_{0}^{\left(n_{k}\right)}=1 / 4, p_{1}^{\left(n_{k}\right)}=3 / 4$. We define the measure $\mu$ by the rule that $\mu\left(I_{\omega}\right)=p_{\omega_{1}}^{(1)} p_{\omega_{2}}^{(2)} \cdots p_{\omega_{n}}^{(n)}$ for $\omega=\left(\omega_{1}, \ldots, \omega_{n}\right)$. The support of $\mu$ is $C$. Provided $\left(n_{k}\right)$ is sufficiently sparse, it can be verified that

$$
\begin{gathered}
\operatorname{dim}_{\mathrm{A}} \operatorname{supp} \mu=\operatorname{dim}_{\mathrm{qA}} \operatorname{supp} \mu=\operatorname{dim}_{\mathrm{H}} C=\log 2 / \log 3, \\
1=\frac{-\log p_{0}^{(n)}}{\log 3}=\operatorname{dim}_{\mathrm{qA}} \mu
\end{gathered}
$$

and

$$
\log 4 / \log 3=-\log \left(\liminf p_{0}^{\left(n_{k}\right)}\right) / \log 3=\operatorname{dim}_{\mathrm{A}} \mu .
$$

If instead $p_{0}^{\left(n_{k}\right)}=1 / k$, then $\operatorname{dim}_{\mathrm{A}} \mu=\infty$. The details are left to the reader.

(ii) A measure $\mu$ satisfying $\operatorname{dim}_{\mathrm{A}} \operatorname{supp} \mu>\operatorname{dim}_{\mathrm{qA}} \mu$ : By taking as $\mu$ the uniform Cantor measure on a Cantor set $C$ with suitably varying ratios of dissection, we can arrange for

$$
\operatorname{dim}_{\mathrm{qA}} \operatorname{supp} \mu=\log 2 / \log 3=\operatorname{dim}_{\mathrm{qA}} \mu<\operatorname{dim}_{\mathrm{A}} \operatorname{supp} \mu=\operatorname{dim}_{\mathrm{A}} \mu .
$$

(iii) By varying both the probabilities and ratios we can construct measures $\mu$ satisfying

$$
\begin{gathered}
\operatorname{dim}_{\mathrm{qA}} \operatorname{supp} \mu<\operatorname{dim}_{\mathrm{A}} \operatorname{supp} \mu<\operatorname{dim}_{\mathrm{qA}} \mu<\operatorname{dim}_{\mathrm{A}} \mu \text { or } \\
\operatorname{dim}_{\mathrm{qA}} \operatorname{supp} \mu<\operatorname{dim}_{\mathrm{qA}} \mu<\operatorname{dim}_{\mathrm{A}} \operatorname{supp} \mu<\operatorname{dim}_{\mathrm{A}} \mu .
\end{gathered}
$$

In [7, Proposition 3.1] it was observed that the Assouad dimension of a measure is finite if and only if the measure is doubling. In Example 5.11 we show that a measure can fail to be doubling, but have finite quasi-Assouad dimension. In Section 4 we characterize finite quasi-Assouad dimension in terms of a weaker doubling-type condition.

Recall that the upper local dimension of a Borel probability measure $\mu$ at $x \in \operatorname{supp} \mu$ is

$$
\overline{\operatorname{dim}}_{\text {loc }} \mu(x)=\limsup _{r \rightarrow 0} \frac{\log \mu(B(x, r))}{\log r} .
$$


The lower local dimension, denoted $\underline{\operatorname{dim}}_{\mathrm{loc}} \mu(x)$, is defined analogously, replacing lim sup by lim inf. If the upper and lower local dimensions coincide, their common value is known as the local dimension of $\mu$ at $x$ and we write $\operatorname{dim}_{\text {loc }} \mu(x)$.

The Assouad dimension is bounded below by the upper local dimension for any point in the support of $\mu$, see [7]. The same holds for the quasi-Assouad dimension.

Proposition 2.4. Let $\mu$ be a Borel probability measure on $\mathbb{R}^{d}$. Then

$$
\operatorname{dim}_{\mathrm{qA}} \mu \geq \sup \left\{\overline{\operatorname{dim}}_{\text {loc }} \mu(x): x \in \operatorname{supp} \mu\right\} .
$$

Proof. Let $s=\sup \left\{\overline{\operatorname{dim}}_{\text {loc }} \mu(x): x \in \operatorname{supp} \mu\right\}$. Temporarily fix $\tau>0$. We will show the quasi-Assouad dimension of $\mu$ is at least $s-\tau$.

To begin the proof, fix $\delta>0$, choose $0<\varepsilon<\tau / 2$ so small that $\delta(\tau-2 \varepsilon)>3 \varepsilon$ and select $x \in \operatorname{supp} \mu$ such that $\overline{\operatorname{dim}}_{\text {loc }} \mu(x) \geq s-\varepsilon$. Choose a decreasing sequence $R_{n} \rightarrow 0$ such that $R_{n+1} \leq R_{n}^{1+\delta}$ and satisfying the property

for all $n$. Then

$$
s-2 \varepsilon \leq \frac{\log \mu\left(B\left(x, R_{n}\right)\right)}{\log R_{n}} \leq s+\varepsilon
$$

$$
\frac{\mu\left(B\left(x, R_{n}\right)\right)}{\mu\left(B\left(x, R_{n+1}\right)\right)} \geq \frac{R_{n}^{s+\varepsilon}}{R_{n+1}^{s-2 \varepsilon}} .
$$

The choice of $\varepsilon$ ensures that $R_{n+1}^{\tau-2 \varepsilon} \leq R_{n}^{(1+\delta)(\tau-2 \varepsilon)} \leq R_{n}^{\tau+\varepsilon}$ and therefore

$$
\frac{R_{n}^{s+\varepsilon}}{R_{n+1}^{s-2 \varepsilon}} \geq\left(\frac{R_{n}}{R_{n+1}}\right)^{s-\tau}
$$

That implies $\operatorname{dim}_{\mathrm{qA}} \mu \geq s-\tau$, as claimed, and as $\tau$ was arbitrary the desired conclusion holds.

Note that the measure of Example 2.3(i) has its supremal local dimension occuring at 0 and this value coincides with the quasi-Assouad (but not the Assouad) dimension of the measure. In Theorem 5.8 we exhibit a class of measures for which this continues to be true. However, Example 3.2 shows that it is also possible for the quasi-Assouad dimension to be strictly larger.

\section{Self-similar Iterated Function Systems}

3.1. Iterated function systems and separation conditions. Let $\left\{S_{j}\right\}$ be a finite family of contractions on $\mathbb{R}$ such that $S_{j}(x)=r_{j} x+d_{j}, j=0, \ldots, m-1$, where $r_{j}>0$. Let $\left(p_{0}, \ldots, p_{m-1}\right)$ be a non-degenerate probability vector, i.e. $p_{j}>0$ and $\sum_{j=0}^{m-1} p_{j}=1$. We refer to the collection $\left\{S_{j}\right\}$ as an iterated function system (IFS) and the collection of tuples $\left\{S_{j}, p_{j}\right\}$ as a weighted iterated function system.

There exists a unique non-empty compact set satisfying $F=\bigcup_{j=0}^{m-1} S_{j}(F)$, called the attractor, associated with the collection $S_{j}$. As all maps are similarities the attractor is known as a self-similar set. Throughout this article we will assume that the self-similar set is the unit line $[0,1]$. There is no loss of generality in assuming $S_{0}(0)=0$ and $S_{m-1}(1)=1$ as $r_{j}>0$.

We can similarly define a unique Borel probability measure by assigning weights to the maps. The resulting measure has support $F$. In fact, this measure is simply the projection of a Bernoulli measure from the underlying symbolic dynamics onto $\mathbb{R}$ and is referred to 
as the self-similar measure associated with the weighted iterated function system $\left\{S_{j}, p_{j}\right\}$. More precisely, the self-similar measure is the unique probability measure $\mu$ satisfying

$$
\mu(E)=\sum_{j=0}^{m-1} p_{j} \mu\left(S_{j}^{-1}(E)\right) \text { for all Borel sets } E .
$$

We let

$$
\lambda=\min r_{j}
$$

and write $\Omega$ for the set of finite words on the alphabet $\{0,1, \ldots, m-1\}$. If all $r_{j}$ are equal, we say that the iterated function system is equicontractive. Given a (finite) word $w=$ $\left(w_{1}, \ldots, w_{n}\right)$, we let $w^{-}=\left(w_{1}, \ldots, w_{n-1}\right)$ and denote the length of $w$ by $|w|$. We usually write words by concatenation, that is $w=w_{1} w_{2} w_{3} \cdots$, and we define

$$
r_{w}=r_{w_{1}} r_{w_{2}} \cdots r_{w_{n}} \text { and } p_{w}=p_{w_{1}} p_{w_{2}} \cdots p_{w_{n}} .
$$

Commonly, separation conditions are employed to give precise results about these attractors and measures. If $F$ is the attractor of the IFS $\left\{S_{i}\right\}$ and $S_{i}(F) \cap S_{j}(F)=\varnothing$ for all $i \neq j$, we say that the IFS satisfies the strong separation condition (SSC). If there is an open set $U$ such that $S_{i}(U) \subseteq U$ for all $i \in \Lambda$ and $S_{i}(U) \cap S_{j}(U)=\varnothing$ for all $i \neq j$, we say that IFS $\left\{S_{i}\right\}$ satisfies the open set condition (OSC). The OSC is a less restrictive condition than the SSC, but in both cases the Hausdorff and Assouad dimensions coincide for the attractor and their common value is given by the unique $s$ that satisfies $\sum r_{i}^{s}=1$, see [5, Cor. 2.11].

Fraser and Howroyd proved that in the case when self-similar measures $\mu$ satisfy the strong separation condition, the Assouad dimension coincides with the supremal local dimension of $\mu$, 7, Theorem 2.4]. Since the quasi-Assouad dimension falls between these two we immediately have the following.

Proposition 3.1. If $\mu$ is a self-similar measure satisfying the strong separation condition, then

$$
\operatorname{dim}_{A} \mu=\operatorname{dim}_{q A} \mu=\sup _{x}\left\{\overline{\operatorname{dim}}_{l o c} \mu(x)\right\} .
$$

This, however, can fail when relaxing the condition to the open set condition as the following example shows.

Example 3.2. A self-similar measure satisfying the OSC, with $\operatorname{dim}_{q A} \mu>\sup _{x}\left\{\overline{\operatorname{dim}}_{l o c} \mu(x)\right\}$.

Consider the IFS $S_{0}(x)=x / 2$ and $S_{1}(x)=x / 2+1 / 2$, with probabilities $p_{0}>p_{1}$. Although the self-similar set of the IFS is $[0,1]$, the open set condition is satisfied with the open set $U=(0,1)$. Temporarily fix $\delta>0$. Choose $N$ large and let $k=\lfloor\delta N\rfloor$. Take $x$ to be the midpoint of the interval $S_{01^{[N+k]}}[0,1]$, where $1^{[N+k]}$ is the word consisting of $N+k$ many letters 1 . This interval has left endpoint $1 / 2$ and length $2^{-(N+k)}$. Choose $R=2^{-N}$ and $r=2^{-(N+k+2)}$, so $r \leq R^{1+\delta}$. Then $B(x, R)$ contains $S_{1^{[N]}[0,1]}$, so $\mu(B(x, R)) \geq p_{1} p_{0}^{N}$, while $B(x, r) \subseteq S_{01^{[N+k]}}[0,1]$ and hence has $\mu$-measure at most $p_{0} p_{1}^{N+k}$. Thus if we are to have

$$
\frac{\mu(B(x, R))}{\mu(B(x, r))} \leq C_{1}\left(\frac{R}{r}\right)^{s}
$$

for all large $N$, it must be true that $2^{\delta s} \geq p_{0} p_{1}^{-(1+\delta)}$, in other words,

$$
s \geq \log 2\left(\frac{1}{\delta}+1\right)\left|\log p_{1}\right|-\frac{\left|\log p_{0}\right|}{\delta}=\left|\log p_{1}\right|+\frac{\left|\log p_{1}\right|-\left|\log p_{0}\right|}{\delta} .
$$


This inequality shows that $H(\delta) \rightarrow \infty$ as $\delta \rightarrow 0$, hence $\operatorname{dim}_{\mathrm{qA}} \mu$ is infinite.

As this IFS satisfies the open set condition, it is known that

$$
\left\{\overline{\operatorname{dim}}_{\text {loc }} \mu(x): x \in \operatorname{supp} \mu\right\}=\left[\frac{\left|\log p_{0}\right|}{\log 2}, \frac{\left|\log p_{1}\right|}{\log 2}\right],
$$

thus the inequality of Proposition 2.4 can be strict.

In this article we will focus on the quasi-Assouad dimension and will show that various desirable properties hold under even weaker conditions that we will now state.

Recall that $\lambda=\min r_{j}$. Set

$$
\Lambda_{n}=\left\{u \in \Omega: r_{u} \leq \lambda^{n} \text { and } r_{u^{-}}>\lambda^{n}\right\}
$$

for the set of words that are comparable to $\lambda^{n}$. In the equicontractive setting, $\Lambda_{n}$ are simply the words of length $n$.

Lau and Ngai [15] studied self-similar IFS under a weaker separation condition that limits the number of overlapping distinct images. This so-called weak separation condition subsequently turned out to be the 'correct' separation condition to consider when dealing with the Assouad dimension of sets. Indeed, the Assouad dimension coincides with the Hausdorff dimension for self-similar sets satisfying the weak separation condition and is maximal otherwise, see [6].

We now recall this definition. Let

$$
\mathcal{A}(x, r)=\left\{v \in \Omega:\left|S_{v}([0,1])\right| \leq r,\left|S_{v^{-}}([0,1])\right|>r \text { and } x \in S_{v}([0,1])\right\}
$$

and

$$
\mathcal{M}(x, r)=\left\{S_{v}: v \in \mathcal{A}(x, r)\right\} .
$$

The self-similar IFS $\left\{S_{i}\right\}$ is said to satisfy the weak separation condition if

$$
\sup _{r \in(0,1)} \sup _{x \in[0,1]} \# \mathcal{M}(x, r)<\infty .
$$

Zerner 23] showed that this is equivalent to the identity not being an accummulation point of

$$
\mathcal{E}=\left\{S_{v}^{-1} \circ S_{w}: v, w \in \Omega\right\}
$$

with respect to the pointwise topology.

Iterated function systems generating Bernoulli convolutions where the contraction ratio is the reciprocal of a Pisot number, as well as iterated function systems of the form $\left(S_{j}\right)$ where $S_{j}(x)=x / d+d_{j}$ where $d \in \mathbb{N}$ and $d_{j} \in \mathbb{Q}$ are examples that satisfy the WSC.

Using Zerner's definition, it is straightforward to show that Definition 3.3 below is another equivalent way of stating the weak separation condition. We have opted to state it in this version as this is the form that we will use in this article.

Definition 3.3. An iterated function system $\left\{S_{j}\right\}$ satisfies the weak separation condition (WSC) if there exists $a>0$ such that if $u, w \in \Lambda_{n}$ and $S_{u}(0) \neq S_{w}(0)$, then

$$
\left|S_{u}(0)-S_{w}(0)\right| \geq a \lambda^{n} \quad \text { and } \quad\left|S_{u}(1)-S_{w}(1)\right| \geq a \lambda^{n} .
$$

Motivated by Ngai and Lau's original definition of the weak separation condition, Feng [4] introduced a separation condition, known as the asymptotic weak separation condition, also in terms of overlapping images. 
Definition 3.4. Let $\left\{S_{i}\right\}$ be a self-similar IFS. We say that $\left\{S_{i}\right\}$ satisfies the asymptotic weak separation condition (AWSC) if there exists non-decreasing function $g(r)$ such that

$$
\log g(r) / \log r \rightarrow 0 \quad \text { and } \quad \sup _{x \in[0,1]} \# \mathcal{M}(x, r) \leq g(r) .
$$

It is easily observed that the AWSC is weaker than the WSC. In a similar fashion, we define a useful separation condition on the asymptotic separation of images of 0 and 1 .

Definition 3.5. An iterated function system $\left\{S_{j}\right\}$ satisfies the asymptotic gap weak separation condition $(\boldsymbol{A} \boldsymbol{G} \boldsymbol{W S C})$ if there exists some non-increasing function $f(n)>0$ such that $(\log f(n)) / n \rightarrow 0$ as $n \rightarrow \infty$ and

$\left|S_{u}(0)-S_{w}(0)\right| \geq f(n) \lambda^{n} \quad$ and $\quad\left|S_{u}(1)-S_{w}(1)\right| \geq f(n) \lambda^{n}$

whenever $u, w \in \Lambda_{n}$ and $S_{u}(0) \neq S_{w}(0)$.

Note that the AGWSC is similar in spirit to the AWSC by allowing the defining feature to vary on a subexponential scale rather than be finite. We will show below that the AGWSC implies the AWSC. While closely related, we are not able to show that these two conditions are equivalent. Note, however, that the two notions coincide in the only known family to satisfy the AWSC (or AGWSC), but not the WSC. This family are the IFSs generating Bernoulli convolutions with contraction ratio the inverse of Salem numbers, see [4].

Lemma 3.6. Let $\left\{S_{i}\right\}$ be a self-similar IFS of the unit line that satisfies the AGWSC. Then $\left\{S_{i}\right\}$ satisfies the $A W S C$.

Proof. Let $x_{0} \in[0,1]$ and let $v \in \Omega$ be such that $x_{0} \in S_{v}([0,1])$. Set $r=\left|S_{v}([0,1])\right|$, then any element $S \in \mathcal{M}\left(x_{0}, r\right)$ satisfies $\gamma r<|S([0,1])| \leq r$ for some uniform $\gamma>0$. Thus

$$
S(0), S(1) \in\left[S_{v}(0)-r, S_{v}(0)+2 r\right] .
$$

Now, since the IFS satisfies the AGWSC, no two distinct maps $S_{1}, S_{2} \in \mathcal{M}\left(x_{0}, r\right)$ may have both $\left|S_{1}(0)-S_{2}(0)\right|<\lambda^{n} \cdot f(n)$ and $\left|S_{1}(1)-S_{2}(1)\right|<\lambda^{n} \cdot f(n)$, where $n$ is such that $r \leq \lambda^{n+1}$ and $r>\lambda^{n+2}$. Thus, there are at most

$$
\frac{3 r}{\lambda^{n} f(n)} \leq \frac{3}{\lambda f(n)}
$$

choices for $S(0)$ and $S(1)$, giving

$$
\# \mathcal{M}\left(x_{0}, r\right) \leq\left(\frac{3}{\lambda f(n)}\right)^{2}
$$

Now $v$, and thus $r$, was arbitrary and so the above inequality holds for all $n$. We obtain

$$
\lim _{r \rightarrow 0} \frac{\# \mathcal{M}\left(x_{0}, r\right)}{|\log r|} \leq \lim _{n \rightarrow \infty} \frac{2 \log 3-2 \log (\lambda f(n))}{\left|\log \lambda^{n+2}\right|}=0,
$$

showing that the AWSC is satisfied.

Since we will fix a self-similar measure by fixing a weighted iterated function system, we will also refer to a measure $\mu$ as satisfying the AGWSC or WSC, where we should say "the weighted iterated function system associated with $\mu$ ".

In the weak separation case we may assume without loss of generality that the constant $a$ arising in the definition of the weak separation condition satisfies $a<\lambda$. Similarly, in the asymptotic gap weak separation case we can assume that $f(n)<\lambda$ for all $n$. 
Definition 3.7. Let $\left\{S_{i}\right\}$ be an equicontractive iterated function system with contraction ratio $\lambda$. Further, let $c>0$ be such that $c(1-\lambda)$ is the diameter of the associated attractor. The IFS is said to be of finite type if there is a finite set $F \subseteq \mathbb{R}$ such that if $u, w \in \Lambda_{n}$ then either

$$
\left|S_{u}(0)-S_{w}(0)\right|>c \lambda^{n} \quad \text { or } \quad \lambda^{-n}\left(S_{u}(0)-S_{w}(0)\right) \in F .
$$

The notion of finite type was introduced by Ngai and Wang [18. It can also be defined for IFS that are not equicontractive, but as this is more technical and not needed in this article, we omit its definition. Examples of iterated function systems of finite type include Bernoulli convolutions with contraction ratio the inverse of Pisot numbers. For further information, the interested reader may peruse [12] and the references cited therein.

We have the following inclusions among these classes, all of which are known to be proper except for the last:

$$
\text { OSC } \subset \text { Finite Type } \subset \text { WSC } \subset \text { AGWSC } \subseteq \text { AWSC } .
$$

3.2. Net intervals. Recall that the attractor of the IFS is assumed to be $[0,1]$. For each $n \in \mathbb{N}$, let $h_{1}, \ldots, h_{s_{n}}$ denote the elements of the set $\left\{S_{u}(0), S_{u}(1): u \in \Lambda_{n}\right\}$, listed in increasing order. Put

$$
\mathcal{F}_{n}=\left\{\left[h_{j}, h_{j+1}\right]: 1 \leq j \leq s_{n}-1\right\} .
$$

The elements of $\mathcal{F}_{n}$ are called the net intervals of level $n$. Of course, the net intervals of a given level $n \geq 1$ cover $[0,1]$. The interval $[0,1]$ will be the (unique) net interval of level 0 . Since $\left|S_{u}(0)-S_{u}(1)\right| \leq r_{u} \leq \lambda^{n}$ when $u \in \Lambda_{n}$, any net interval of level $n$ has length at most $\lambda^{n}$. We denote the length of the net interval $\Delta$ by $l(\Delta)$.

Each $x \in[0,1]$ belongs to either one or two net intervals of level $n$. The point $x$ will belong to two net intervals if and only if $x$ is an endpoint of a net interval of level $n$. In both cases we refer to the net interval of level $n$ containing $x$ by $\Delta_{n}(x)$, choosing arbitrarily when it is not unique. Each net interval $\Delta$ of level $n$ is contained in a unique net interval of level $n-1$ which we refer to as the parent of $\Delta$.

Given a net interval $\Delta$ of level $n$, let

$$
P_{n}(\Delta)=\sum_{\substack{u \in \Lambda_{n} \\ S_{u}[0,1] \supseteq \underline{D} \Delta}} p_{u} .
$$

Note that $P_{n}(\Delta) \geq \min p_{j}^{n}$. Since $P_{n}(\Delta)$ is the sum of all weights of words whose images cover the net interval $\Delta$, we must have $P_{n}\left(\Delta_{n}(x)\right) \geq \mu\left(\Delta_{n}(x)\right)$. As $l\left(\Delta_{n}(x)\right) \leq \lambda^{n}$, the ball $B\left(x, \lambda^{n}\right)$ contains $\Delta_{n}(x)$. In particular, if $u \in \Lambda_{n}$ and $x \in S_{u}([0,1])$, then $S_{u}([0,1]) \subseteq$ $B\left(x, \lambda^{n}\right)$. Thus,

$$
\mu\left(B\left(x, \lambda^{n}\right)\right) \geq P_{n}\left(\Delta_{n}(x)\right) \geq \mu\left(\Delta_{n}(x)\right) .
$$

To compare the minimal and maximal contraction rate we define $\Theta \in \mathbb{N}$ implicitly as the least integer satisfying

$$
\left(\max _{j \in \Lambda} r_{j}\right)^{\Theta+1}<\lambda^{2}=\left(\min _{j \in \Lambda} r_{j}\right)^{2}
$$

We collect some further properties of $P_{n}(\Delta)$ below.

Lemma 3.8. Let $\Theta$ be as above. Then

(a) $P_{n}\left(\Delta_{n}(x)\right) \geq \min p_{j}^{\Theta} P_{n-1}\left(\Delta_{n-1}(x)\right)$ and 
(b) $P_{n}\left(\Delta_{n}(x)\right) \leq P_{n-1}\left(\Delta_{n-1}(x)\right)$.

Proof. (a) Let $u \in \Lambda_{n-1}$ and suppose $S_{u}[0,1] \supseteq \Delta_{n-1}(x)$. Then there exists some word $w$ such that $u w \in \Lambda_{n}$ and $S_{u w}[0,1] \supseteq \Delta_{n}(x)$. Note that $r_{w^{-}}>\lambda$ and so $r_{w} \geq \lambda^{2}$. Using the definition of $\Theta$ we find that $|w| \leq \Theta$. As $p_{u}=p_{u w} p_{w}^{-1}$, we have

$$
P_{n-1}\left(\Delta_{n-1}(x)\right) \leq P_{n}\left(\Delta_{n}(x)\right)\left(\min p_{w}\right)^{-1} \leq P_{n}\left(\Delta_{n}(x)\right)\left(\min p_{j}^{\Theta}\right)^{-1} .
$$

(b) Suppose $v \in \Lambda_{n}$ and $S_{v}[0,1] \supseteq \Delta_{n}(x)$. Then $v=u w$ where $u \in \Lambda_{n-1}$ and $S_{u}[0,1] \supseteq$ $\Delta_{n-1}(x)$. Furthermore, the sum of $p_{w}$ taken over such $w$ is at most one. Thus

$$
P_{n}\left(\Delta_{n}(x)\right)=\sum_{\substack{u w \in \Lambda_{n}, u \in \Lambda_{n-1} \\ S_{u w}[0,1] \supseteq \Delta_{n}(x)}} p_{u} p_{w} \leq P_{n-1}\left(\Delta_{n-1}(x)\right) .
$$

Now suppose the iterated function system satisfies the asymptotic gap weak separation condition with function $f(n)$. For each $n$, choose the minimal integer $\kappa_{n}$ such that $\lambda^{\kappa_{n}} \leq$ $f(n)$. By definition,

$$
\kappa_{n} \leq \frac{\log f(n)}{\log \lambda}+1
$$

and therefore $\kappa_{n} / n \rightarrow 0$. As $\lambda^{n+\kappa_{n}} \leq f(n) \lambda^{n}$,

$$
B\left(x, \lambda^{n+\kappa_{n}}\right) \subseteq B\left(x, f(n) \lambda^{n}\right) .
$$

If $u \in \Lambda_{n+\kappa_{n}}$, then $r_{u} \leq \lambda^{n+\kappa_{n}}$. If also $S_{u}[0,1] \supseteq \Delta_{n+\kappa_{n}}(x)$, then $S_{u}[0,1] \subseteq B\left(x, f(n) \lambda^{n}\right)$, and so by (3.1),

$$
\mu\left(B\left(x, f(n) \lambda^{n}\right)\right) \geq P_{n+\kappa_{n}}\left(\Delta_{n+\kappa_{n}}(x)\right) .
$$

If $u \in \Lambda_{n}$ and $S_{u}(0)=0$, then $S_{u}(1)=r_{u} \geq \lambda^{n+1} \geq f(n) \lambda_{n}$, hence $l\left(\Delta_{n}(0)\right) \geq f(n) \lambda^{n}$. Consequently,

$$
B\left(0, f(n) \lambda^{n}\right) \cap[0,1] \subseteq \Delta_{n}(0) \subseteq B\left(0, \lambda^{n}\right) .
$$

Combining these observations with the previous lemma gives the following bounds.

Proposition 3.9. Suppose $\mu$ is a self-similar measure that satisfies the asymptotic gap weak separation condition with function $f(n)$. There is a constant $0<A<1$ such that for any $x, n$

$$
A^{\kappa_{n}} P_{n}\left(\Delta_{n}(x)\right) \leq \mu\left(B\left(x, f(n) \lambda^{n}\right)\right) .
$$

In particular,

$$
A^{\kappa_{n}} P_{n}\left(\Delta_{n}(0)\right) \leq \mu\left(B\left(0, f(n) \lambda^{n}\right)\right) \leq \mu\left(\Delta_{n}(0)\right) \leq P_{n}\left(\Delta_{n}(0)\right) .
$$

Proof. Lemma 3.8(a) implies $P_{n+\kappa_{n}}\left(\Delta_{n+\kappa_{n}}(x)\right) \geq A^{\kappa_{n}} P_{n}\left(\Delta_{n}(x)\right)$ for $A=\min p_{j}^{\Theta}$. This fact, coupled with (3.4), gives the first statement. The second statement follows similarly from (3.2) and (3.5).

Let $s, t$ be

$$
s=\liminf \left(P_{n}\left(\Delta_{n}(0)\right)^{1 / n} \quad \text { and } \quad t=\liminf \left(P_{n}\left(\Delta_{n}(1)\right)^{1 / n} .\right.\right.
$$

Note that $s, t>0$ since $P_{n}\left(\Delta_{n}(x)\right) \geq\left(\min _{j} p_{j}\right)^{n}$ for all $x, n$. 
Proposition 3.10. Suppose $\mu$ is a self-similar measure that satisfies the asymptotic gap weak separation condition. Then

$$
\overline{\operatorname{dim}}_{\text {loc }} \mu(0)=\frac{\log s}{\log \lambda} \quad \text { and } \quad \overline{\operatorname{dim}}_{\text {loc }} \mu(1)=\frac{\log t}{\log \lambda} .
$$

Furthermore, $s, t<1$.

Proof. Our earlier observations show that

$$
\begin{aligned}
B\left(0, \lambda_{n}^{n+\kappa_{n}}\right) \cap[0,1] & \subseteq B\left(0, f(n) \lambda^{n}\right) \cap[0,1] \subseteq \Delta_{n}(0) \subseteq B\left(0, \lambda^{n}\right) \\
& \subseteq B\left(0, f\left(n-\kappa_{n}\right) \lambda^{n-\kappa_{n}}\right) \cap[0,1] \subseteq \Delta_{n-\kappa_{n}}(0),
\end{aligned}
$$

and by Proposition 3.9.

$$
\begin{aligned}
A^{\kappa_{n}} P_{n}\left(\Delta_{n}(0)\right) & \leq \mu\left(B\left(0, \lambda^{n}\right)\right) \leq \mu\left(\Delta_{n-\kappa_{n}}(0)\right) \\
& \leq P_{n-\kappa_{n}}\left(\Delta_{n-\kappa_{n}}(0)\right) \leq A^{-\kappa_{n}} P_{n}\left(\Delta_{n}(0)\right) .
\end{aligned}
$$

Since $\kappa_{n} / n \rightarrow 0$ as $n \rightarrow \infty$,

$$
\begin{aligned}
\limsup _{n \rightarrow \infty} \frac{\log \left(P_{n}\left(\Delta_{n}(0)\right)\right)}{n \log \lambda} \leq \limsup _{n \rightarrow \infty} \frac{-\kappa_{n} \log A+\log \left(P_{n}\left(\Delta_{n}(0)\right)\right)}{n \log \lambda} \leq \limsup _{n \rightarrow \infty} \frac{\log \left(\mu\left(B\left(0, \lambda^{n}\right)\right)\right)}{n \log \lambda} \\
=\varlimsup_{\operatorname{dim}} \mu(0) \leq \limsup _{n \rightarrow \infty} \frac{\kappa_{n} \log A+\log \left(P_{n}\left(\Delta_{n}(0)\right)\right)}{n \log \lambda} \leq \limsup _{n \rightarrow \infty} \frac{\log \left(P_{n}\left(\Delta_{n}(0)\right)\right)}{n \log \lambda} .
\end{aligned}
$$

This proves the first equality. The second equality follows similarly, and is omitted for brevity.

To prove that $s<1$, note that there must be at least one index $j$ such that $S_{j}(0) \neq 0$. Without loss of generality assume the index is $j_{0}$. Thus, if $\tau \in \Lambda_{n}$ with $S_{\tau}[0,1] \supseteq \Delta_{n}(0)$, and $\tau=u w$ where $u \in \Lambda_{n-1}$ and $S_{u}[0,1] \supseteq \Delta_{n-1}(0)$, then $w$ does not contain the letter $j_{0}$. It follows that

$$
P_{n}\left(\Delta_{n}(0)\right) \leq\left(1-p_{j_{0}}\right) \sum_{\substack{u \in \Lambda_{n-1} \\ S_{u}[0,1] \supseteq \Delta_{n-1}}} p_{u}=\left(1-p_{j_{0}}\right) P_{n-1}\left(\Delta_{n-1}(0)\right)
$$

and hence $P_{n}\left(\Delta_{n}(0)\right) \leq\left(1-p_{j_{0}}\right)^{n}$. Thus $s \leq 1-p_{j_{0}}<1$. The case for $t<1$ follows along the same lines and is omitted.

\section{Measures With Finite quasi-Assouad Dimension}

In [7. Proposition 3.1] it was proven that the Assouad dimension of a measure is finite if and only if the measure is doubling. This is not required for finite quasi-Assouad dimension, as is shown in Example 5.11.

In this section we characterize the measures with finite quasi-Assouad dimension that satisfy the asymptotic gap weak separation condition in terms of a weak doubling-like condition. This characterization is stated in Corollary 4.6 .

Definition 4.1. A compactly supported Borel probability measure $\mu$ is quasi-doubling if for every $\varepsilon>0$ and positive, non-decreasing function $G(r)$ satisfying $\log G(r) / \log r \rightarrow 0$ as $r \rightarrow 0$, there exists $c>0$ such that

$$
\mu(B(x, G(r) r)) \geq c r^{\varepsilon} \mu(B(x, 2 r))
$$

for all $x \in \operatorname{supp} \mu$ and $r \in(0,1)$. 
By considering $r=\lambda^{n}$ for any (fixed) $\lambda<1$ and $g(n)=G\left(\lambda^{n}\right)$, one can check that this definition is equivalent to the statement that $\mu$ is quasi-doubling if and only if for every $q>1$, constant $b>0$ and positive, non-increasing sequence $(g(n))_{n}$ satisfying $\log g(n) / n \rightarrow 0$, there exists $c>0$ such that

$$
\mu\left(B\left(x, g(n) \lambda^{n}\right)\right) \geq c q^{-n} \mu\left(B\left(x, b \lambda^{n}\right)\right),
$$

for all $x \in \operatorname{supp} \mu$ and $n \in \mathbb{N}$. We will often use the definition of quasi-doubling in this formulation, or for a particular choice of $b$, which is also equivalent.

We first check that doubling implies quasi-doubling and that quasi-doubling is necessary for finite quasi-Assouad dimension.

Proposition 4.2. (i) If $\mu$ is a doubling measure, then $\mu$ is quasi-doubling.

(ii) If $\operatorname{dim}_{q A} \mu<\infty$, then $\mu$ is quasi-doubling.

Proof. (i) Pick $\lambda<1$ assume the constant $C$ is chosen so that $\mu(B(x, \lambda r)) \geq C \mu(B(x, r))$ for all $x, r$. Fix $q>1$ and choose $\varepsilon>0$ so that $C^{\varepsilon}>q^{-1}$. Let $(g(n))_{n}$ be a non-increasing sequence satisfying $\log g(n) / n \rightarrow 0$. As $\log g(n) / n \rightarrow 0$ there is some $n_{0}$ such that $g(n) \geq \lambda^{\varepsilon n}$ for all $n \geq n_{0}$. Since $g$ is non-increasing, $g(n) \geq g\left(n_{0}\right) \geq \lambda^{\varepsilon n_{0}} \geq A \lambda^{\varepsilon n}$ for all $n \leq n_{0}$ and a suitable constant $A$. By repeated application of the doubling property,

$$
\mu\left(B\left(x, g(n) \lambda^{n}\right)\right) \geq \mu\left(B\left(x, A \lambda^{\varepsilon n} \lambda^{n}\right)\right) \geq C^{n \varepsilon+1} \mu\left(B\left(x, A \lambda^{n}\right)\right) \geq C q^{-n} \mu\left(B\left(x, A \lambda^{n}\right)\right)
$$

and this gives 4.2.

(ii) Suppose $\operatorname{dim}_{\mathrm{qA}} \mu<t<\infty$ and let $q>1>\lambda$. Select $\delta>0$ so that $\lambda^{-\delta t}=q$. The definition of the quasi-Assouad dimension ensures that for some $C>0$,

$$
\frac{\mu\left(B\left(x, \lambda^{n}\right)\right)}{\mu\left(B\left(x, \lambda^{n(1+\delta)}\right)\right)} \leq C \lambda^{-n \delta t}=C q^{n} .
$$

Assume $\log g(n) / n \rightarrow 0$. Then $g(n) \geq \lambda^{\delta n}$ for $n$ sufficiently large. Hence $B\left(x, \lambda^{n(1+\delta)}\right) \subseteq$ $B\left(x, g(n) \lambda^{n}\right)$ and therefore

$$
C^{-1} q^{-n} \mu\left(B\left(x, \lambda^{n}\right)\right) \leq \mu\left(B\left(x, \lambda^{n(1+\delta)}\right)\right) \leq \mu\left(B\left(x, g(n) \lambda^{n}\right)\right)
$$

for sufficiently large $n$ and this suffices to show $\mu$ is quasi-doubling.

Before stating our main result of this section, we introduce further notation for self-similar measures $\mu$ with support $[0,1]$ and minimal contraction factor $\lambda$.

Given a level $n$ net interval, $\Delta_{n}$, other than $\Delta_{n}(1)$ or $\Delta_{n}(0)$, we let $\Delta_{n}^{R}$ be the union of the two net intervals of level $n$ immediately to the right of $\Delta_{n}$, and let $\Delta_{n}^{L}$ be the union of the two net intervals immediately to the left, with the understanding that $\Delta_{n}^{R}=\Delta_{n}(1)$ if $\Delta_{n}$ is immediately adjacent to $\Delta_{n}(1)$ and similarly for $\Delta_{n}^{L}$. If $\Delta_{n}=\Delta_{n}(1)$ we only define $\Delta_{n}^{L}$ and if $\Delta_{n}=\Delta_{n}(0)$ we only define $\Delta_{n}^{R}$.

We remark that if the IFS associated with $\mu$ satisfies the asymptotic gap weak separation condition with function $f(n)$, any net interval whose endpoints are $S_{u}(0)$ and $S_{w}(0)$ for some $u, w \in \Lambda_{n}$ has length at least $f(n) \lambda^{n}$ and likewise if the endpoints are both iterates of 1 . Consequently, the length of the union of any two adjacent net intervals has length at least $f(n) \lambda^{n}$. In particular, this is true for $\Delta_{n}^{R}$ and $\Delta_{n}^{L}$ since $\Delta_{n}(0)$ and $\Delta_{n}(1)$ also have length at least $f(n) \lambda^{n}$. 
Definition 4.3. A self-similar measure $\mu$ that satisfies the asymptotic gap weak separation condition with function $f(n)$ is quasi-net doubling if for every $q>1$ there exist $c_{1}, c_{2}>0$ such that if $l\left(\Delta_{n}\right) \geq f(n+1) \lambda^{n+1}$, then

$$
c_{1} q^{-n} \mu\left(\Delta_{n}^{*}\right) \leq \mu\left(\Delta_{n}\right) \leq c_{2} q^{n} \mu\left(\Delta_{n}^{*}\right)
$$

for $\Delta_{n}^{*}=\Delta_{n}^{L}$ and $\Delta_{n}^{*}=\Delta_{n}^{R}$, where defined.

Theorem 4.4. Suppose the self-similar measure $\mu$ has support $[0,1]$ and satisfies the asymptotic gap weak separation condition. Then $\operatorname{dim}_{\mathrm{qA}} \mu<\infty$ if and only if $\mu$ is quasi-net doubling.

Proof. Suppose $\mu$ satisfies the AGWSC with function $f(n)$ and has finite quasi-Assouad dimension. By Prop. 4.2(ii) $\mu$ is quasi-doubling and, in particular, satisfies (4.2) for all $q>1$ with $g(n)=f(n+1) \lambda / 2$ and $b=3$. We will see that this already implies $\mu$ is quasi-net doubling. In other words, we will prove that if there is a constant $c$ so that

$$
\mu\left(B\left(x, f(n+1) \lambda^{n+1} / 2\right)\right) \geq c q^{-n} \mu\left(B\left(x, 3 \lambda^{n}\right)\right)
$$

for all $x \in \operatorname{supp} \mu$ and positive integers $n$, then $\mu$ is quasi-net doubling.

Assume $\Delta_{n}$ is a level $n$ net interval with $l\left(\Delta_{n}\right) \geq f(n+1) \lambda^{n+1}$. Let $z$ be the midpoint of $\Delta_{n}$ and let $\Delta_{n}^{*}$ refer to either $\Delta_{n}^{L}$ or $\Delta_{n}^{R}$. Then $B\left(z, f(n+1) \lambda^{n+1} / 2\right) \subseteq \Delta_{n}$ and $B\left(z, 3 \lambda^{n}\right) \supseteq$ $\Delta_{n}^{*}$. Thus, using (4.2),

$$
\mu\left(\Delta_{n}\right) \geq \mu\left(B\left(z, f(n+1) \lambda^{n+1} / 2\right)\right) \geq c q^{-n} \mu\left(B\left(z, 3 \lambda^{n}\right)\right) \geq c q^{-n} \mu\left(\Delta_{n}^{*}\right) .
$$

This proves the left hand inequality in (4.4).

Similarly, we can prove the other inequality. Recall that $l\left(\Delta_{n}^{*}\right) \geq f(n) \lambda^{n}$ and so, letting $z$ be the midpoint of $\Delta_{n}^{*}$, and using (4.2), we obtain

$$
\mu\left(\Delta_{n}^{*}\right) \geq \mu\left(B\left(z, f(n) \lambda^{n}\right)\right) \geq c q^{-n} \mu\left(B\left(z, 3 \lambda^{n}\right)\right) \geq c q^{-n} \mu\left(\Delta_{n}\right) .
$$

This proves the right hand inequality and therefore $\mu$ is quasi-net doubling.

Now assume $\mu$ is quasi-net doubling and denote by $\lambda$ the minimal contraction factor. Without loss of generality we can assume $\lambda<1 / 2$, for if not, we can replace the IFS $\left\{S_{j}\right\}$ with suitable $k$-fold compositions of the maps $S_{j}$.

Fix $\delta>0$ and let $N_{0}$ be large enough such that

$$
f(N+1) \geq \lambda^{N \delta / 2}
$$

for all $N \geq N_{0}$. Such an $N_{0}$ exists as the asymptotic gap weak separation condition guarantees $\log (f(n)) / n \rightarrow 0$ as $n \rightarrow 0$. We will be using the bounds of (4.4) with $q=2^{\delta /(1+\delta)}>1$ and consider $\Delta_{N}(x)$.

Case 1: Assume that $l\left(\Delta_{N}(x)\right) \geq f(N+1) \lambda^{N+1}$. As $\Delta_{N}^{R}$ and $\Delta_{N}^{L}$ have length at least $f(N) \lambda^{N}$

$$
B\left(x, f(N)(1-\lambda) \lambda^{N}\right) \cap[0,1] \subseteq \Delta_{N}(x) \cup \Delta_{N}^{R} \cup \Delta_{N}^{L}
$$

and thus by the quasi-net doubling condition,

$$
\begin{aligned}
\mu\left(B\left(x, f(N)(1-\lambda) \lambda^{N}\right)\right) & \leq \mu\left(\Delta_{N}(x)\right)+\mu\left(\Delta_{N}^{R}\right)+\mu\left(\Delta_{N}^{L}\right) \\
& \leq c q^{N} \mu\left(\Delta_{N}(x)\right)
\end{aligned}
$$


for some $c>0$. From (3.2), we have $\mu\left(\Delta_{N}(x)\right) \leq P_{N}\left(\Delta_{N}(x)\right)$ and $\mu\left(B\left(x, \lambda^{n}\right)\right) \geq P_{n}\left(\Delta_{n}(x)\right)$ for any $n$. Let $t=\left(\min p_{j}\right)^{-\Theta}$, where $\Theta$ is given in (3.3). It now follows from Lemma 3.8 that

$$
\frac{\mu\left(B\left(x, f(N)(1-\lambda) \lambda^{N}\right)\right)}{\mu\left(B\left(x, 3 \lambda^{n}\right)\right)} \leq \frac{c q^{N} P_{N}\left(\Delta_{N}(x)\right)}{P_{n}\left(\Delta_{n}(x)\right)} \leq c q^{N} t^{n-N} .
$$

The "gap" between $r$ and $R$ in the definition of the quasi-Assouad dimension means that we can restrict our attention to the case where

$$
\lambda^{n} \leq\left(f(N)(1-\lambda) \lambda^{N}\right)^{1+\delta} .
$$

We can therefore assume without loss of generality that $n \geq N\left(1+\delta^{\prime}\right)$ for all $\delta^{\prime}<\delta$. In particular, this holds for $\delta^{\prime}=\delta /(1+\delta)$. Rearranging gives $N \delta /(1+\delta)=N \delta^{\prime} \leq(n-N)$ and hence $q^{N}=2^{N \delta^{\prime}} \leq 2^{n-N}$. Taking $\beta=\log 2 t /|\log \lambda|$ we have

$$
\frac{\mu\left(B\left(x, f(N)(1-\lambda) \lambda^{N}\right)\right)}{\mu\left(B\left(x, 3 \lambda^{n}\right)\right)} \leq c \lambda^{-\beta(n-N)} .
$$

Using (4.6), we have

$$
\frac{f(N+1)(1-\lambda) \lambda^{N}}{3 \lambda^{n}} \geq c_{1} \lambda^{N-n} \lambda^{N \delta / 2} \geq c_{1} \lambda^{-(n-N) / 2},
$$

for all $n \geq\left(1+\delta^{\prime}\right) N>N \geq N_{0}$ and some $c_{1}>0$. Redefining $c$, if necessary, we obtain

$$
\frac{\mu\left(B\left(x, f(N)(1-\lambda) \lambda^{N}\right)\right)}{\mu\left(B\left(x, 3 \lambda^{n}\right)\right)} \leq c\left(\frac{f(N+1)(1-\lambda) \lambda^{N}}{3 \lambda^{n}}\right)^{2 \beta} .
$$

We next show that in the second case we obtain the same bound, before establishing that this is sufficient to guarantee finite quasi-Assouad dimension.

Case 2: Assume $l\left(\Delta_{N}(x)\right)<f(N+1) \lambda^{N+1}$. In this case, $\Delta_{N}(x)$ cannot contain two net subintervals of level $N+1$ as their union would have length at least $f(N+1) \lambda^{N+1}$. Thus $\Delta_{N+1}(x)=\Delta_{N}(x)$. Fix $n$ such that $3 \lambda^{n} \leq\left(f(N)(1-\lambda) \lambda^{N}\right)^{1+\delta}$ and choose the maximal integer $j$ such that $N<j \leq n$ and $\Delta_{N}(x)=\cdots=\Delta_{j}(x)$.

Since the union of two adjacent level $N$ net intervals has length at least $f(N) \lambda^{N}$, it follows that the level $N$ net intervals immediately adjacent to $\Delta_{N}(x)$ have length at least $f(N)(1-\lambda) \lambda^{N}$. Denote the left and right net intervals of $\Delta_{N}(x)$ by $\Delta_{N}^{r}$ and $\Delta_{N}^{l}$ respectively. Thus

$$
B\left(x, f(N)(1-\lambda) \lambda^{N}\right) \cap[0,1] \subseteq \Delta_{N}(x) \cup \Delta_{N}^{r} \cup \Delta_{N}^{l} .
$$

Let $x_{1}, x_{2}$ be the midpoints of $\Delta_{j}^{r}$ and $\Delta_{j}^{l}$ respectively. As each level $j$ net interval has length at most $\lambda^{j}$ we have $B\left(x_{i}, \lambda^{j}\right) \subseteq B\left(x, 3 \lambda^{j}\right)$ for $i=1,2$.

These observations yield the bounds

$$
\begin{aligned}
\mu\left(B\left(x, 3 \lambda^{j}\right)\right) & \geq \max \left(\mu\left(B\left(x, \lambda^{j}\right)\right), \mu\left(B\left(x_{1}, \lambda^{j}\right)\right), \mu\left(B\left(x_{2}, \lambda^{j}\right)\right)\right) \\
& \geq \max \left(P_{j}\left(\Delta_{j}(x)\right), P_{j}\left(\Delta_{j}^{r}\right), P_{j}\left(\Delta_{j}^{l}\right)\right)
\end{aligned}
$$

and

$$
\begin{aligned}
\mu\left(B\left(x, f(N)(1-\lambda) \lambda^{N}\right)\right) & \leq 3 \max \left(\mu\left(\Delta_{N}(x)\right), \mu\left(\Delta_{N}^{r}\right), \mu\left(\Delta_{N}^{l}\right)\right) \\
& \leq 3 \max \left(P_{N}\left(\Delta_{N}(x)\right), P_{N}\left(\Delta_{N}^{r}\right), P_{N}\left(\Delta_{N}^{l}\right)\right) .
\end{aligned}
$$


Since $\Delta_{N}(x)=\Delta_{j}(x)$, it follows that $\Delta_{j}^{r} \subseteq \Delta_{N}^{r}$ and $\Delta_{j}^{l} \subseteq \Delta_{N}^{l}$, so

$$
\frac{\mu\left(B\left(x, f(N)(1-\lambda) \lambda^{N}\right)\right)}{\mu\left(B\left(x, 3 \lambda^{j}\right)\right)} \leq 3 \max \left(\frac{P_{N}\left(\Delta_{N}(x)\right)}{P_{j}\left(\Delta_{j}(x)\right)}, \frac{P_{N}\left(\Delta_{N}^{r}\right)}{P_{j}\left(\Delta_{j}^{r}\right)}, \frac{\left.P_{N}\left(\Delta_{N}^{l}\right)\right)}{\left.P_{j}\left(\Delta_{j}^{l}\right)\right)}\right) \leq 3 t^{j-N},
$$

where $t=\left(\min p_{j}\right)^{-\Theta}$. If $j=n$, then as in Case 1 , we have

$$
\frac{\mu\left(B\left(x, f(N)(1-\lambda) \lambda^{N}\right)\right)}{\mu\left(B\left(x, 3 \lambda^{n}\right)\right)} \leq c\left(\frac{f(N+1)(1-\lambda) \lambda^{N}}{3 \lambda^{n}}\right)^{2 \beta} .
$$

Otherwise, $j<n$ so that $\Delta_{j+1}(x) \neq \Delta_{j}(x)$. That ensures $\Delta_{j}(x)$ contains at least two $(j+1)$-level net intervals and so its length is at least $f(j+1) \lambda^{j+1}$. Thus the quasi-net doubling condition implies

$$
\mu\left(\Delta_{j}(x)\right)+\mu\left(\Delta_{j}^{R}\right)+\mu\left(\Delta_{j}^{L}\right) \leq c q^{j} \mu\left(\Delta_{j}(x)\right) \leq c q^{j} P_{j}\left(\Delta_{j}(x)\right)
$$

and hence

$$
\frac{\mu\left(\Delta_{j}(x) \cup \Delta_{j}^{R} \cup \Delta_{j}^{L}\right)}{\mu\left(B\left(x, 3 \lambda^{n}\right)\right)} \leq c q^{j} \frac{P_{j}\left(\Delta_{j}(x)\right)}{P_{n}\left(\Delta_{n}(x)\right)} \leq c q^{j} t^{n-j} .
$$

We will deal with the case where the maximum of $P_{N}\left(\Delta_{N}(x)\right), P_{N}\left(\Delta_{N}^{r}\right)$, and $P_{N}\left(\Delta_{N}^{l}\right)$ is $P_{N}\left(\Delta_{N}^{r}\right)$. The other two cases are analogous and left to the reader.

Let $y_{1}$ be the right endpoint of $\Delta_{j}(x)$. Then

$$
B\left(y_{1}, f(j+1) \lambda^{j+1}\right) \cap[0,1] \subseteq \Delta_{j}(x) \cup \Delta_{j}^{R} \cup \Delta_{j}^{L},
$$

so applying (3.4) we have

$$
\mu\left(\Delta_{j}(x)\right)+\mu\left(\Delta_{j}^{R}\right)+\mu\left(\Delta_{j}^{L}\right) \geq \mu\left(B\left(y_{1}, f(j+1) \lambda^{j+1}\right) \geq P_{j+1+\kappa_{j+1}}\left(\Delta_{j+1+\kappa_{j+1}}\left(y_{1}\right)\right) .\right.
$$

where we are free to take $\Delta_{j+1+\kappa_{j+1}}\left(y_{1}\right)$ to be the net interval having $y_{1}$ as the left endpoint. But as $\Delta_{j}(x)=\Delta_{N}(x), y_{1}$ is also the left endpoint of $\Delta_{N}^{r}$. So we can choose $\Delta_{N}\left(y_{1}\right)=\Delta_{N}^{r}$. Combining these observations gives

$$
\begin{aligned}
\frac{\mu\left(B\left(x, f(N)(1-\lambda) \lambda^{N}\right)\right)}{\mu\left(\Delta_{j}(x) \cup \Delta_{j}^{R} \cup \Delta_{j}^{L}\right)} & \leq \frac{3 P_{N}\left(\Delta_{N}^{r}\right)}{P_{j+1+\kappa_{j}}\left(\Delta_{j+1+\kappa_{j+1}}\left(y_{1}\right)\right)} \\
& \leq \frac{3 P_{N}\left(\Delta_{N}\left(y_{1}\right)\right)}{P_{j+1+\kappa_{j+1}}\left(\Delta_{j+1+\kappa_{j+1}}\left(y_{1}\right)\right)} \leq c t^{j+\kappa_{j+1}-N} .
\end{aligned}
$$

Consequently,

$$
\begin{aligned}
\frac{\mu\left(B\left(x, f(N)(1-\lambda) \lambda^{N}\right)\right)}{\mu\left(B\left(x, 3 \lambda^{n}\right)\right)} & \leq \frac{\mu\left(B\left(x, f(N)(1-\lambda) \lambda^{N}\right)\right)}{\mu\left(\Delta_{j}(x) \cup \Delta_{j}^{R} \cup \Delta_{j}^{L}\right)} \frac{\mu\left(\Delta_{j}(x) \cup \Delta_{j}^{R} \cup \Delta_{j}^{L}\right)}{\mu\left(B\left(x, 3 \lambda^{n}\right)\right)} \\
& \leq c t^{j+\kappa_{j+1}+1-N} q^{j} t^{n-j} \leq c q^{n} t^{n-N+\kappa_{n}} \leq c 2^{n-N} t^{n-N+\kappa_{n}} .
\end{aligned}
$$

Since $(\log f(N+1)) / N$ and $\kappa_{n} / n$ tend to zero for increasing $n, N$, there exists $N_{1}$ such that

$$
\left(1-\frac{N}{n}\right) \log \left(2 t \lambda^{2 \beta}\right) \leq 2 \beta \frac{\log f(N+1)}{n}-\frac{\kappa_{n}}{n} \log t
$$

for all $n \geq(1+\delta) N \geq N_{1}$. Thus

$$
(2 t)^{n-N} t^{\kappa_{n}} \leq\left(f(N+1) \lambda^{-(n-N)}\right)^{2 \beta}
$$


and that ensures

$$
\frac{\mu\left(B\left(x, f(N)(1-\lambda) \lambda^{N}\right)\right)}{\mu\left(B\left(x, 3 \lambda^{n}\right)\right)} \leq c\left(\frac{f(N+1)(1-\lambda) \lambda^{N}}{3 \lambda^{n}}\right)^{2 \beta}
$$

for all $N \geq N_{1}$.

Having established the same upper bound in (4.7), 4.8), and (4.9) it remains to show that this is sufficient for the quasi-Assouad dimension to be finite. Let $N_{2}=\max \left\{N_{0}, N_{1}\right\}$. For fixed $\delta^{\prime}>\delta$, let $r \leq R^{1+\delta^{\prime}} \leq R<f\left(N_{2}\right)(1-\lambda) \lambda^{N_{2}}$ and choose $N \geq N_{2}$ and $n$ such that

$$
f(N+1)(1-\lambda) \lambda^{N+1} \leq R \leq f(N)(1-\lambda) \lambda^{N} \quad \text { and } \quad 3 \lambda^{n} \leq r \leq 3 \lambda^{n-1} .
$$

We note that this is well-defined as $f$ is non-increasing and $r \leq R^{1+\delta^{\prime}}$ gives $n \geq(1+\delta) N$. Now, by appealing to (4.7), (4.8), and (4.9), we have

$$
\frac{\mu(B(x, R))}{\mu(B(x, r))} \leq \frac{\mu\left(B\left(x, f(N)(1-\lambda) \lambda^{N}\right)\right)}{\mu\left(B\left(x, 3 \lambda^{n}\right)\right)} \leq c\left(\frac{f(N+1)(1-\lambda) \lambda^{N}}{3 \lambda^{n}}\right)^{2 \beta} \leq c\left(\frac{R}{r}\right)^{2 \beta} .
$$

This proves that $\operatorname{dim}_{\mathrm{qA}} \mu \leq 2 \beta<\infty$.

We finish this section by providing a classification of the quasi-Assouad dimension in terms of quasi-doubling.

Corollary 4.5. Let $\mu$ be a self-similar measure that satisfies the asymptotic gap weak separation condition and with $\operatorname{supp} \mu=[0,1]$. Then $\operatorname{dim}_{\mathrm{qA}} \mu<\infty$ if and only if $\mu$ is quasi-doubling.

Proof. The first part of the proof of Theorem 4.4 actually shows that quasi-doubling implies quasi-net doubling and thus has finite quasi-Assouad dimension.

Corollary 4.6. Let $\mu$ be a self-similar measure with $\operatorname{supp} \mu=[0,1]$. Suppose $\mu$ satisfies the weak separation condition with minimal contraction factor $\lambda$. Then $\operatorname{dim}_{\mathrm{qA}} \mu<\infty$ if and only if for every $q>1$ and $0<A<B$ there exist constants $c=c(q, A, B)$ such that

$$
\mu\left(B\left(x, A \lambda^{n}\right)\right) \geq c q^{-n} \mu\left(B\left(x, B \lambda^{n}\right)\right)
$$

for all $n \in \mathbb{N}$ and $x \in \operatorname{supp} \mu$. Equivalently, $\operatorname{dim}_{\mathrm{qA}} \mu<\infty$ if and only if for every $\varepsilon>0$, there is a constant $C$ such that

$$
\mu(B(x, r)) \geq C r^{\varepsilon} \mu(B(x, 2 r))
$$

for all $r \in(0,1)$ and $x \in \operatorname{supp} \mu$.

Note that this Corollary includes Theorem A.

Proof. As we observe in (4.5), satisfying (4.10) with $A=a \lambda / 2$ for $a$ the constant arising in the definition of the WSC and $B=3$ is enough to ensure the measure is quasi-net doubling and hence has finite Assouad dimension.

\section{Dimensions of WEAKLY COMPARABLE AND GENERALIZED REgUlar MEASURES}

The equicontractive self-similar measure $\mu$ arising from the weighted IFS, $\left\{S_{j}, p_{j}\right\}$, is said to be regular if $p_{0}=p_{m-1}=\min p_{j}$. In this section we study the more general classes of generalized regular and weakly comparable measures with the goal of proving Theorem $\mathrm{B}$. 


\subsection{Weakly comparable measures.}

Definition 5.1. A self-similar measure $\mu$ with support $[0,1]$ is weakly comparable if for each $q>1$ there is a constant $c$, depending on $q$, such that for all $n$ and adjacent net intervals $\Delta_{1}, \Delta_{2}$ of level $n$, we have

$$
\frac{1}{c} q^{-n} P_{n}\left(\Delta_{2}\right) \leq P_{n}\left(\Delta_{1}\right) \leq c q^{n} P_{n}\left(\Delta_{2}\right) .
$$

Weakly comparable measures were originally introduced in [12] where they were called comparable measures. Property (5.1) is very useful for studying dimensional properties since it allows one to approximate the (often poorly understood) quantities $\mu\left(B\left(x, \lambda^{n}\right)\right)$ by the (often better understood) $P_{n}(\Delta)$ for $\Delta$ containing $x$.

In Corollary 5.6 we will show that any regular self-similar measure is weakly comparable. If an IFS is of finite type, then the net intervals of level $n$ are comparable in size, thus if a finite type measure is doubling, then it is weakly comparable. On the other hand, Example 5.11 gives an equicontractive, finite type IFS that is regular, hence weakly comparable, but not doubling and therefore has infinite Assouad dimension.

However, weakly comparable measures have finite quasi-Assouad dimension, as we see next.

Proposition 5.2. If $\mu$ is a weakly comparable, self-similar measure that satisfies the asymptotic gap weak separation condition, then $\mu$ is quasi-net doubling and hence has finite quasi-Assouad dimension.

Proof. Fix $q_{0}>1$ and let $q=\left(q_{0}\right)^{1 / 3}>1$. By Lemma 3.8 there is a constant $A=\min p_{j}^{\Theta}$ such that $P_{n}\left(\Delta_{n}(x)\right) \geq A P_{n-1}\left(\Delta_{n-1}(x)\right)$ for all $n$ and $x \in[0,1]$. Choose $\varepsilon>0$ so that $A^{\varepsilon} \geq q^{-1}$. Given any net interval $\Delta_{n}(x)$ we have

$$
\mu\left(\Delta_{n}^{*}\right) \leq P_{n}\left(\Delta_{n}^{L}\right)+P_{n}\left(\Delta_{n}^{R}\right) \leq c q^{2 n} P_{n}\left(\Delta_{n}(x)\right),
$$

where $\Delta_{n}^{*}, \Delta_{n}^{L}, \Delta_{n}^{R}$ are as in Definition 4.3 . Note that by the definition of quasi-net doubling we only need to check the case when $l\left(\Delta_{n}(x)\right) \geq f(n+1) \lambda^{n+1}$. Taking $z$ to be the midpoint of $\Delta_{n}(x)$ we have

$$
\Delta_{n}(x) \supseteq B\left(z, f(n+1) \lambda^{n+1} / 2\right) \supseteq B\left(z, f(n+2) \lambda^{n+2}\right),
$$

hence Lemma 3.8 and (3.4) yield

$$
\mu\left(\Delta_{n}(x)\right) \geq \mu\left(B\left(z, f(n+2) \lambda^{n+2}\right)\right) \geq P_{n+2+\kappa_{n+2}}\left(\Delta_{n+2+\kappa_{n+2}}(x)\right) \geq A^{2+\kappa_{n+2}} P_{n}\left(\Delta_{n}(x)\right) .
$$

For large enough $n$, the weakly comparable assumption thus implies

$$
\begin{aligned}
\mu\left(\Delta_{n}(x)\right) & \geq A^{2} A^{n \varepsilon} P_{n}\left(\Delta_{n}(x)\right) \geq A^{2} q^{-n} P_{n}\left(\Delta_{n}(x)\right) \\
& \geq A^{2} q^{-3 n} c^{-1} \mu\left(\Delta_{n}^{*}\right) \geq A^{2} q_{0}^{-n} c^{-1} \mu\left(\Delta_{n}^{*}\right) .
\end{aligned}
$$

The inequality $\mu\left(\Delta_{n}^{*}\right) \geq c_{2} q_{0}^{-n} \mu\left(\Delta_{n}(x)\right)$ follows analogously. This shows that $\mu$ is quasinet doubling.

The next result is similar in spirit, but more technical, and will be used later to find upper bounds on the quasi-Assouad dimension. 
Lemma 5.3. Suppose $\mu$ is a weakly comparable, self-similar measure that satisfies the asymptotic gap weak separation condition with function $f(n)$. Then for any $q>1$ there are constants $c_{1}, c_{2}>0$ depending on $q$ such that, for all $x \in[0,1]$ and $n \in \mathbb{N}$,

$$
c_{1} q^{-n} P_{n}\left(\Delta_{n}(x)\right) \leq P_{n+\kappa_{n}}\left(\Delta_{n+\kappa_{n}}(x)\right) \leq \mu\left(B\left(x, f(n) \lambda^{n}\right)\right) \leq c_{2} q^{n} P_{n}\left(\Delta_{n}(x)\right) .
$$

Proof. Since $l\left(\Delta_{n}^{*}\right) \geq f(n) \lambda^{n}$, we have

$$
B\left(x, f(n) \lambda^{n}\right) \cap[0,1] \subseteq \Delta_{n}(x) \cup \Delta_{n}^{R} \cup \Delta_{n}^{L},
$$

so that $\mu\left(B\left(x, f(n) \lambda^{n}\right)\right) \leq c q^{n} P_{n}\left(\Delta_{n}(x)\right)$ for some $c>0$. Similar reasoning to the above shows that $P_{n+\kappa_{n}}\left(\Delta_{n+\kappa_{n}}(x)\right) \geq q^{-n} P_{n}\left(\Delta_{n}(x)\right)$ for $n$ sufficiently large and as we always have $P_{n+\kappa_{n}}\left(\Delta_{n+\kappa_{n}}(x)\right) \leq \mu\left(B\left(x, f(n) \lambda^{n}\right)\right)$, the inequalities of (5.2) are complete.

5.2. Generalized regular measures. To define generalized regular measures, we first need to introduce further terminology. We assume $\operatorname{supp} \mu=[0,1]$.

Suppose $\Delta \in \mathcal{F}_{N}$ has descendent net subinterval $\Delta^{\prime} \in \mathcal{F}_{N+n}$. If $u \in \Lambda_{N}$ with $S_{u}[0,1] \supseteq \Delta$, then there is some word $w$ such that $u w \in \Lambda_{n+N}$ and $S_{u w}[0,1] \supseteq \Delta^{\prime}$. We call such a word $w$ a path of level $n$ (of $\Delta)$. Clearly,

$$
P_{N+n}\left(\Delta_{N+n}(x)\right) \geq \inf \left\{p_{w}: w \text { path of level } n\right\} P_{N}\left(\Delta_{N}(x)\right) .
$$

We call $w$ a left-edge path if $S_{w}(0)=0$ and a right-edge path if $S_{w}(1)=1$. Put

$$
\Gamma_{\Delta, n}^{L}=\sum_{\begin{array}{c}
w \text { left-edge path } \\
\text { of } \Delta \text { of level } n
\end{array}} p_{w} \quad \text { and } \quad \Gamma_{n}^{L}=\Gamma_{[0,1], n}^{L} .
$$

We define $\Gamma_{\Delta, n}^{R}$ and $\Gamma_{n}^{R}$ similarly, and set

$$
\Gamma_{n}=\Gamma_{n}^{L}+\Gamma_{n}^{R} \text { and } \Gamma_{\Delta, n}=\Gamma_{\Delta, n}^{R}+\Gamma_{\Delta, n}^{L} .
$$

Note that

$$
\Gamma_{n}^{L}=\sum_{\substack{w \in \Lambda_{n} \\ S_{w}(0)=0}} p_{w}=P_{n}\left(\Delta_{n}(0)\right)
$$

hence the constants $s, t$ introduced in (3.6) are also equal to

$$
s=\liminf _{n \rightarrow \infty}\left(\Gamma_{n}^{L}\right)^{1 / n}, t=\liminf _{n \rightarrow \infty}\left(\Gamma_{n}^{R}\right)^{1 / n} .
$$

For each positive integer $n$ and $x \in[0,1]$, let

$$
Q_{n}(x)=\sup _{N \in \mathbb{N}} \frac{P_{N}\left(\Delta_{N}(x)\right)}{P_{N+n}\left(\Delta_{N+n}(x)\right)}
$$

where $\Delta_{N+n}(x)$ is a child of $\Delta_{N}(x)$ containing $x$ (with $P_{N+n}\left(\Delta_{N+n}(x)\right.$ ) minimal if there are two choices). Set

$$
Q_{n}=\sup _{x \in \operatorname{supp} \mu} Q_{n}(x) .
$$

Of course, $Q_{n} \leq Q_{1}^{n}$ and by Lemma 3.8, $Q_{1} \leq\left(\min _{j} p_{j}\right)^{-\Theta}$ where $\Theta$ is given by (3.3).

Definition 5.4. The weighted iterated function system $\left\{S_{j}, p_{j}\right\}$ is generalized regular if for each $q>1$,

$$
\lim _{n \rightarrow \infty} Q_{n} q^{-n} \sup _{\Delta} \Gamma_{\Delta, n}=0
$$

where the supremum is taken over all net intervals. 
We will also call the self-similar measure $\mu$ associated with $\left\{S_{j}, p_{j}\right\}$ a generalized regular measure.

In order to show that regular measures are generalized regular, we first prove that all $\Gamma_{\Delta, n}$ are comparable to $\Gamma_{n}$.

Proposition 5.5. There exists $c>0$ such that $\Gamma_{n}^{L} \leq \Gamma_{\Delta, n}^{L} \leq c \Gamma_{n}^{L}$ for all $n$ and $\Delta$. Similarly, $\Gamma_{n}^{R}$ is comparable to $\Gamma_{\Delta, n}^{R}$.

Proof. Let $\Delta=[a, b]$ be a net interval of level $N$. Then $\Gamma_{\Delta, n}^{L}=\sum p_{w}$, where the sum is over all $w$ where $S_{w}(0)=0$ and there is some $u \in \Lambda_{N}$ such that $u w \in \Lambda_{N+n}$ and $S_{u}(0)=a$. This means that $w, u$ must satisfy the conditions $r_{u} \leq \lambda^{N}, r_{u^{-}}>\lambda^{N}, r_{u w} \leq \lambda^{N+n}$ and $r_{(u w)^{-}}=r_{u w^{-}}>\lambda^{N+n}$. Since $r_{j} \geq \lambda$ for all $j$, it follows that $r_{w}<\lambda^{n-1}$ and $r_{w^{-}}>\lambda^{n}$. Consequently,

$$
\begin{aligned}
\Gamma_{\Delta, n}^{L} \leq & \sum_{\substack{S_{w}(0)=0, r_{w}<\lambda^{n-1}, r_{w^{-}}>\lambda^{n}}} p_{w} \\
= & \sum_{\substack{S_{w}(0)=0, r_{w} \leq \lambda^{n}, r_{w^{-}}>\lambda^{n}}} p_{w}+\sum_{\substack{S_{w}(0)=0, r_{w} \in\left(\lambda^{n}, \lambda^{n-1}\right), r_{w^{-}}>\lambda^{n}}} p_{w}
\end{aligned}
$$

Recall that $w \in \Lambda_{n}$ is equivalent to $w$ satisfying $r_{w} \leq \lambda^{n}$ and $r_{w^{-}}>\lambda^{n}$, thus the left sum in (5.3) is equal to $\Gamma_{n}^{L}$. For the second sum, let $k$ be the minimal integer such that $r_{0}^{k} \leq \lambda$. For fixed $r_{w} \in\left(\lambda^{n}, \lambda^{n-1}\right)$, choose $j=j(w) \in\{1,2, \ldots, k\}$ such that $r_{w} r_{0}^{j} \leq \lambda^{n}$ and $r_{w} r_{0}^{j-1}>\lambda^{n}$. Let $\overline{0}_{j}$ be the unique word of length $j$ containing just the letter 0 . Then

$$
\begin{aligned}
\sum_{\substack{S_{w}(0)=0, r_{w} \in\left(\lambda^{n}, \lambda^{n-1}\right), r_{w^{-}}>\lambda^{n}}} p_{w} & \leq \sum_{j=1}^{k} \sum_{\substack{S_{w}(0)=0 \\
w \overline{0}_{j} \in \Lambda_{n}}} p_{w}=\sum_{j=1}^{k} p_{0}^{-j} \sum_{\substack{S_{\tau}(0)=0 \\
\tau=w \overline{0}_{j} \in \Lambda_{n}}} p_{\tau} \\
& \leq \sum_{j=1}^{k} p_{0}^{-j} \sum_{\substack{S_{\tau}(0)=0 \\
\tau \in \Lambda_{n}}} p_{\tau} \leq k p_{0}^{-k} \Gamma_{n}^{L} .
\end{aligned}
$$

We conclude $\Gamma_{\Delta, n}^{L} \leq\left(1+k p_{0}^{-k}\right) \Gamma_{n}^{L}$, as required.

Corollary 5.6. (i) A self-similar measure $\mu$ is generalized regular if and only if

$$
\lim _{n} Q_{n} q^{-n} \Gamma_{n}^{L}=\lim _{n} Q_{n} q^{-n} \Gamma_{n}^{R}=0 \quad \text { for every } \quad q>1 .
$$

(ii) An equicontractive, regular self-similar measure $\left(p_{0}=p_{m-1}=\min _{j} p_{j}\right)$ is generalized regular.

Proof. (i) follows immediately from the Proposition.

(ii) The edge paths of level $n$ of such a measure are the words $(0)^{n}$ and $(1)^{n}$, so $\Gamma_{n}=$ $p_{0}^{n}+p_{m-1}^{n}$. Furthermore, $Q_{n} \leq\left(\min p_{j}\right)^{-n}=p_{0}^{-n}=p_{m-1}^{-n}$, hence the measure is generalized regular.

The notion of 'generalized regular' was introduced in the study of non-equicontractive finite type iterated function systems where it was observed that generalized regular implies weakly comparable (see [12, Theorem 4.11]). In fact, this holds in general. 
Proposition 5.7. A generalized regular, self-similar measure $\mu$ is weakly comparable.

Proof. Fix $q>1$ and choose $N_{0}$ so $\sup _{\Delta} \Gamma_{\Delta, n} \leq Q_{n}^{-1} q^{n} / 2$ for all $n \geq N_{0}$. Since $P_{n}(\Delta)$ is finite for all $n$, we can find $c>0$ such that

$$
\frac{1}{c} q^{-k} P_{k}\left(\Delta_{2}\right) \leq P_{k}\left(\Delta_{1}\right) \leq c q^{k} P_{k}\left(\Delta_{2}\right)
$$

whenever $\Delta_{1}, \Delta_{2}$ are adjacent net intervals of level $k$ for all $k=1, \ldots, N_{0}$.

Assume $n \geq N_{0}+1$. We proceed by induction on $n$. Suppose $\Delta_{1}, \Delta_{2}$ are adjacent net intervals of level $n$ where, without loss of generality, $\Delta_{1}$ is to the left of $\Delta_{2}$. If $\widehat{\Delta}_{j}$ is the ancestor of $\Delta_{j}$ at level $n-k$, then $P_{n}\left(\Delta_{j}\right) \sim P_{n-k}\left(\widehat{\Delta}_{j}\right)$, with constants of comparability depending only on $k$. Thus we can assume $\Delta_{1}, \Delta_{2}$ have no common ancestor within $N_{0}$ levels.

For $j=1,2$, let $\widehat{\Delta_{j}}$ be the $\left(n-N_{0}\right)$-level ancestor of $\Delta_{j}$. Let $\mathcal{D}_{1}$ denote the words $u \in \Lambda_{n-N_{0}}$ where $S_{u}[0,1]$ contains $\widehat{\Delta_{1}}$, but not $\widehat{\Delta_{2}}$. Define $\mathcal{D}_{2}$ analogously and let $\mathcal{E}$ denote those $u \in \Lambda_{n-N_{0}}$ where $S_{u}[0,1]$ contains both $\widehat{\Delta_{1}}$ and $\widehat{\Delta_{2}}$.

Consider any $\tau \in \Lambda_{n}$ with $S_{\tau}[0,1]$ covering $\Delta_{1}$. Then $\tau=u w$ where $u \in \Lambda_{n-N_{0}}, S_{u}[0,1]$ contains $\widehat{\Delta_{1}}$ and $w$ is a path of level $N_{0}$ of $\widehat{\Delta_{1}}$. The word $u$ belongs to either $\mathcal{D}_{1}$ or $\mathcal{E}$ and in the former case $w$ is a right edge path. Thus

$$
\begin{aligned}
& P_{n}\left(\Delta_{1}\right)=\sum_{u \in \Lambda_{n-N_{0}}} p_{u w} \\
& w \text { path of level } N_{0} \text { of } \widehat{\Delta_{1}} \\
& S_{u w}[0,1] \supseteq \Delta_{1} \\
& =\sum_{\substack{u \in \mathcal{D}_{1} \\
\text { right edge path of level } N_{0}}} p_{u} p_{w}+\sum_{\substack{u \in \mathcal{E} \\
w \text { path of level } N_{0}}} p_{u} p_{w}
\end{aligned}
$$

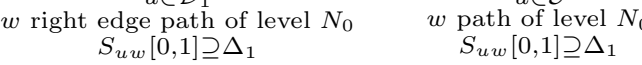

$$
\begin{aligned}
& \leq \sum_{u \in \mathcal{D}_{1}} p_{u} \Gamma_{\widehat{\Delta_{1}}, N_{0}}^{R}+\sum_{u \in \mathcal{E}} p_{u} \\
& \leq \frac{Q_{N_{0}}^{-1} q^{N_{0}}}{2} \sum_{u \in \mathcal{D}_{1}} p_{u}+\sum_{u \in \mathcal{E}} p_{u} .
\end{aligned}
$$

Now $\sum_{u \in \mathcal{D}_{1}} p_{u} \leq \sum_{u \in \mathcal{D}_{1}} p_{u}+\sum_{u \in \mathcal{E}} p_{u}=P_{n-N_{0}}\left(\widehat{\Delta_{1}}\right)$ and $\sum_{u \in \mathcal{E}} p_{u} \leq \sum_{u \in \mathcal{E}} p_{u}+\sum_{u \in \mathcal{D}_{2}} p_{u}=$ $P_{n-N_{0}}\left(\widehat{\Delta_{2}}\right)$. Hence applying the inductive assumption and using the fact that $P_{n-N_{0}}\left(\widehat{\Delta_{2}}\right) \leq$ $P_{n}\left(\Delta_{2}\right) Q_{N_{0}}$ we have

$$
\begin{aligned}
P_{n}\left(\Delta_{1}\right) & \leq \frac{Q_{N_{0}}^{-1} q^{N_{0}}}{2} P_{n-N_{0}}\left(\widehat{\Delta_{1}}\right)+P_{n-N_{0}}\left(\widehat{\Delta_{2}}\right) \\
& \leq\left(\frac{Q_{N_{0}}^{-1} q^{N_{0}}}{2} c q^{n-N_{0}}+1\right) P_{n-N_{0}}\left(\widehat{\Delta_{2}}\right) \\
& \leq\left(\frac{1}{2} Q_{N_{0}}^{-1} c q^{n}+1\right) P_{n}\left(\Delta_{2}\right) Q_{N_{0}} .
\end{aligned}
$$

Taking $c \geq 0$ sufficiently large, we obtain the desired conclusion that $P_{n}\left(\Delta_{1}\right) \leq c q^{n} P_{n}\left(\Delta_{2}\right)$. 
5.3. Dimensions of generalized regular measures. Theorem B is a consequence of Theorem 5.8 below. Other examples of measures satisfying the assumptions of the Theorem below are Bernoulli convolutions with contraction ratios being reciprocals of Salem numbers.

Theorem 5.8. Suppose $\mu$ is a generalized regular, self-similar measure, with support $[0,1]$, that satisfies the asymptotic gap weak separation condition. Then

$$
\operatorname{dim}_{\mathrm{qA}} \mu=\max \left\{\overline{\operatorname{dim}}_{\text {loc }} \mu(0), \overline{\operatorname{dim}}_{\text {loc }} \mu(1)\right\}=\max \left\{\overline{\operatorname{dim}}_{\text {loc }} \mu(x): x \in \operatorname{supp} \mu\right\} .
$$

Corollary 5.9. If $\mu$ is a regular, equicontractive self-similar measure with full support and satisfying the weak separation condition, then

$$
\operatorname{dim}_{\mathrm{qA}} \mu=\max \left\{\overline{\operatorname{dim}}_{\mathrm{loc}} \mu(x): x \in \operatorname{supp} \mu\right\} .
$$

Remark 5.10. In Example 5.5 we see this can fail if the measure does not have full support.

Proof of Theorem 5.8. Without loss of generality, assume $\max \left\{\overline{\operatorname{dim}}_{\text {loc }} \mu(0), \overline{\operatorname{dim}}_{\text {loc }} \mu(1)\right\}=$ $\overline{\operatorname{dim}}_{\text {loc }} \mu(0)$, which by Proposition 3.10 is equal to

$$
d=\log s / \log \lambda \text { where } s=\liminf \left(\Gamma_{n}^{L}\right)^{1 / n} .
$$

First, we will verify that for small enough $\varepsilon>0$ and every $\delta>0$ there are constants $C, C_{0}$, depending on $\varepsilon, \delta$, such that if $r \leq R^{1+\delta} \leq R \leq C_{0}$, then for all $x$,

$$
\frac{\mu(B(x, R))}{\mu(B(x, r))} \leq C\left(\frac{R}{r}\right)^{d(1+\varepsilon)}
$$

Consequently, $\operatorname{dim}_{\mathrm{qA}} \mu \leq d$.

Fix $\delta, \varepsilon>0$ and $x \in[0,1]$. Assume $r \leq R^{1+\delta} \leq R \leq C_{0}$ where $C_{0}$ will be specified later. Choose integers $N, n$ such that $f(N+1) \lambda^{N+1}<R \leq f(N) \lambda^{N}$ and $\lambda^{n} \leq r<\lambda^{n-1}$. By (3.2),

$$
\mu(B(x, r)) \geq \mu\left(B\left(x, \lambda^{n}\right)\right) \geq P_{n}\left(\Delta_{n}(x)\right) .
$$

Since generalized regular measures are weakly comparable,

$$
\mu(B(x, R)) \leq \mu\left(B\left(x, f(N) \lambda^{N}\right)\right) \leq \mathcal{C} q^{N} P_{N}\left(\Delta_{N}(x)\right)
$$

for $\mathcal{C}$ depending on $q$, as per (5.2).

We see that

$$
\frac{\mu(B(x, R))}{\mu(B(x, r))} \leq \frac{\mathcal{C} q^{N} P_{N}\left(\Delta_{N}(x)\right)}{P_{n}\left(\Delta_{n}(x)\right)} \leq \mathcal{C} q^{N} Q_{n-N} .
$$

As $\mu$ is generalized regular, $\Gamma_{m}^{L} Q_{m} q^{-m} \rightarrow 0$ as $m \rightarrow \infty$. Thus, we can choose $N_{1}$ such that if $m=n-N \geq \delta N_{1}$ then $Q_{m} \leq q^{m}\left(\Gamma_{m}^{L}\right)^{-1}$. Therefore

$$
\frac{\mu(B(x, R))}{\mu(B(x, r))} \leq \frac{q^{m} \mathcal{C} q^{N}}{\Gamma_{m}^{L}}=\mathcal{C} q^{n}\left(\Gamma_{m}^{L}\right)^{-1} .
$$

As noted in Proposition 3.10, $s=\liminf \left(\Gamma_{n}^{L}\right)^{1 / n} \in(0,1)$. Thus we can choose $N_{2}$ so that if $m \geq \delta N$ for some $N \geq N_{2}$, then

$$
\Gamma_{m}^{L} \geq s^{m(1+\varepsilon / 2)} .
$$

We require that $C_{0}$ be so small that if $R \leq C_{0}$, then $R \leq f(N) \lambda^{N}$ for $N \geq \max \left(N_{1}, N_{2}\right)$. Hence for $r \leq R^{1+\delta} \leq R \leq C_{0}$ we have

$$
\frac{\mu(B(x, R))}{\mu(B(x, r))} \leq \mathcal{C} q^{n} s^{-m(1+\varepsilon / 2)} .
$$


As $s=\lambda^{d}$, for any fixed $\varepsilon>0$, there exists $c>0$ such that

$$
\left(\frac{R}{r}\right)^{d(1+\varepsilon)} \geq \lambda^{(N-n+2) d(1+\varepsilon)} f(N+1)^{d(1+\varepsilon)}=c f(N+1)^{d(1+\varepsilon)} s^{-m(1+\varepsilon)} .
$$

We note that

$$
\mathcal{C} q^{n} s^{-m(1+\varepsilon / 2)} \leq c f(N+1)^{d(1+\varepsilon)} s^{-m(1+\varepsilon)}
$$

if and only if

$$
C^{\prime} q^{n} f(N+1)^{-d(1+\varepsilon)} \leq s^{-m \varepsilon / 2}
$$

where $C^{\prime}$ is the appropriate constant. Taking logarithms, this is equivalent to

$$
\frac{1}{m}\left(\log C^{\prime}+n \log q-d(1+\varepsilon) \log f(N+1)\right) \leq \varepsilon|\log s| / 2 .
$$

Now, $m=n-N \geq \delta n /(1+\delta)$, so $(n \log q) / m \leq(1+\delta)(\log q) / \delta$ and $(1 / m) \log f(N+1) \rightarrow 0$. Thus with a suitable choice of $q$ close to 1 (depending on $\varepsilon, \delta$ ) and large enough $N$, we can achieve this inequality. With this further constraint on $C_{0}$ it then follows that for a suitable constant $c$, we have

$$
\frac{\mu(B(x, R))}{\mu(B(x, r))} \leq c\left(\frac{R}{r}\right)^{d(1+\varepsilon)} \text { for all } r \leq R^{1+\delta} \leq R \leq C_{0},
$$

and this implies $\operatorname{dim}_{\mathrm{qA}} \mu \leq d(1+\varepsilon)$ for all $\varepsilon>0$. Hence $\operatorname{dim}_{\mathrm{qA}} \mu \leq d$

By Proposition 2.4 we have $\operatorname{dim}_{\mathrm{qA}} \mu \geq \sup \left\{\overline{\operatorname{dim}}_{\text {loc }} \mu(x): x \in \operatorname{supp} \mu\right\} \geq d$, giving the lower bound and hence equality.

In contrast, generalized regular measures satisfying the AGWSC and having full support need not have finite Assouad dimension.

Example 5.11. Consider the IFS $S_{j}(x)=x / 3+d_{j}$ where $d_{0}=0, d_{1}=1 / 6, d_{2}=1 / 3$, $d_{3}=2 / 3$ and probabilities each $1 / 4$. This IFS is equicontractive, finite type, regular and of full support. Thus it is generalized regular and hence weakly comparable. Applying Theorem 5.8 gives $\operatorname{dim}_{q A} \mu=\operatorname{dim}_{l o c} \mu(0)=\log 4 / \log 3$.

However, $\mu$ is not doubling and consequently, the Assouad dimension of $\mu$ is infinite. To see this, one can show that $1 / 2$ is the boundary point of two level $n$ net intervals for each level $n$. The two intervals have the same length, $3^{-n} / 2$. Using the techniques developed in [1] it can be shown that the $\mu$-measure of the right interval is at most $c_{1} 4^{-n}$ for some constant $c_{1}>0$, while the left interval has measure at least $c_{2} n 4^{-n}$ for some $c_{2}>0$. With $R=\left(\frac{3}{4}\right) 3^{-n}, r=\left(\frac{1}{4}\right) 3^{-n}$ and $x_{n}$ the midpoint of the right net interval of level $n$, we have $\mu\left(B\left(x_{n}, R\right)\right) \geq c_{2} n 4^{-n}$ and $\mu\left(B\left(x_{n}, r\right)\right) \leq c_{1} 4^{-n}$, while $R / r=3$, which proves $\mu$ is not doubling.

5.4. Dimensions of weakly comparable measures. For the larger class of weakly comparable measures we can also obtain bounds on the quasi-Assouad dimension. These bounds wil be used to show in Example 5.13 that the generalized regular condition in Theorem 5.8 is not necessary.

Theorem 5.12. Suppose $\mu$ is weakly comparable and satisfies the asymptotic gap weak separation property. Then the quasi-Assouad dimension of $\mu$ is bounded by

$$
\operatorname{dim}_{\mathrm{qA}} \mu \leq \limsup _{n \rightarrow \infty} \frac{-\log Q_{n}}{n \log \lambda}<\infty,
$$


and for all $x \in[0,1]$,

$$
\begin{aligned}
& \overline{\operatorname{dim}}_{\text {loc }} \mu(x)=\limsup _{n \rightarrow \infty} \frac{\log P_{n}\left(\Delta_{n}(x)\right)}{n \log \lambda} \\
& \underline{\operatorname{dim}}_{\text {loc }} \mu(x)=\liminf _{n \rightarrow \infty} \frac{\log P_{n}\left(\Delta_{n}(x)\right)}{n \log \lambda} .
\end{aligned}
$$

Proof. We first prove the upper bound. Let

$$
d=\limsup _{n} \frac{-\log Q_{n}}{n \log \lambda}
$$

It will be enough to prove that $\operatorname{dim}_{\mathrm{qA}} \mu \leq d+\varepsilon$ for all $\varepsilon>0$. Choose $M$ such that

$$
(1 / m) \log Q_{m} \leq-(d+\varepsilon / 2) \log \lambda,
$$

for all $m \geq M$.

Recall that $f(n)$ is decreasing in $n$. Thus, given $r \leq R^{1+\delta} \leq R \leq C_{0}$ we can choose $N$ and $n$ such that

$$
f(N+1) \lambda^{N+1}<R \leq f(N) \lambda^{N}
$$

and

$$
f(n) \lambda^{n} \leq r<f(n-1) \lambda^{n-1}
$$

where $C_{0}$ is chosen sufficiently small to ensure that $N+1 \leq n-1$ and $n-N \geq M$.

Take $q>1$. Then (5.2) yields

$$
\mu(B(x, R)) \leq \mu\left(B\left(x, f(N) \lambda^{N}\right)\right) \leq C q^{N} P_{N}\left(\Delta_{N}(x)\right)
$$

and

$$
\mu(B(x, r)) \geq \mu\left(B\left(x, f(n) \lambda^{n}\right)\right) \geq P_{n+\kappa_{n}}\left(\Delta_{n+\kappa_{n}}(x)\right),
$$

where $C>0$ depends on $q$. Thus

$$
\frac{\mu(B(x, R))}{\mu(B(x, r))} \leq \frac{C q^{N} P_{N}\left(\Delta_{N}(x)\right)}{P_{n+\kappa_{n}}\left(\Delta_{n+\kappa_{n}}(x)\right)} \leq C q^{N} Q_{n+\kappa_{n}-N}(x) .
$$

Using (5.6) and the fact that $\lambda^{\kappa_{n}} \geq \lambda f(n)$, we have

$$
C q^{N} Q_{n+\kappa_{n}-N}(x) \leq C q^{N} \lambda^{-\left(n+\kappa_{n}-N\right)(d+\varepsilon / 2)} \leq C q^{N} \lambda^{-(n-N)(d+\varepsilon / 2)} f(n)^{-(d+\varepsilon / 2)},
$$

redefining $C>0$ as appropriate. Further,

$$
\left(\frac{R}{r}\right)^{d+\varepsilon} \geq\left(\frac{f(N+1)}{f(n-1)}\right)^{d+\varepsilon} \lambda^{(N-n+1)(d+\varepsilon)} \geq c \lambda^{(N-n)(d+\varepsilon)} .
$$

Thus, in order to satisfy

$$
\frac{\mu(B(x, R))}{\mu(B(x, r))} \leq c\left(\frac{R}{r}\right)^{d+\varepsilon}
$$

for some constant $c$, it will be enough to satisfy the inequality

$$
q^{N} \lambda^{-(n-N)(d+\varepsilon / 2)} f(n)^{-(d+\varepsilon / 2)} \leq \lambda^{(N-n)(d+\varepsilon)}
$$

for all $n \geq(1+\delta) N, N$ sufficiently large and for some suitable $q>1$. Equivalently,

$$
(d+\varepsilon / 2) \frac{|\log f(n)|}{n} \leq \frac{(n-N) \varepsilon}{2 n}|\log \lambda|-\frac{N}{n} \log q .
$$


Since $N / n \leq(1+\delta)^{-1}$, the right hand side of the latter expression dominates

$$
\left(1-\frac{1}{1+\delta}\right) \frac{\varepsilon}{2}|\log \lambda|-\frac{1}{1+\delta} \log q
$$

and this is at least

$$
\left(1-\frac{1}{1+\delta}\right) \frac{\varepsilon}{4}|\log \lambda|
$$

if we choose $q$ close enough to 1 . Since $\log f(n) / n \rightarrow 0$ as $n \rightarrow \infty$, we can ensure that this quantity dominates $(d+\varepsilon / 2)|\log f(n)| / n$ for large enough $n$. Suitably redefining $C_{0}>0$, if necessary, will guarantee that $n \geq(1+\delta) N$ is sufficiently large to be sure this is true. From these inequalities it follows that the quasi-Assouad dimension of $\mu$ is at most $d+\varepsilon$ as required.

We now turn to proving the equalities involving the local dimension. For each $q>1$ we have, by (5.2),

$$
\left(C q^{n}\right)^{-1} P_{n}\left(\Delta_{n}(x)\right) \leq \mu\left(B\left(x, f(n) \lambda^{n}\right)\right) \leq C q^{n} P_{n}\left(\Delta_{n}(x)\right)
$$

where $C>0$ depends on $q$. Thus

$$
\begin{aligned}
\frac{\log q}{\log \lambda}+\liminf _{n \rightarrow \infty} \frac{\log P_{n}\left(\Delta_{n}(x)\right)}{n \log \lambda} & \leq \liminf _{n \rightarrow \infty} \frac{\log \mu\left(B\left(x, f(n) \lambda^{n}\right)\right)}{\log \lambda^{n}} \\
& \leq \liminf _{n \rightarrow \infty} \frac{\log P_{n}\left(\Delta_{n}(x)\right)}{n \log \lambda}-\frac{\log q}{\log \lambda}
\end{aligned}
$$

Since the inequality above holds for all $q>1$, we deduce

$$
\liminf _{n \rightarrow \infty} \frac{\log \mu\left(B\left(x, f(n) \lambda^{n}\right)\right)}{\log \lambda^{n}}=\liminf _{n \rightarrow \infty} \frac{\log P_{n}\left(\Delta_{n}(x)\right)}{n \log \lambda}
$$

Given any $r>0$, choose $n$ such that $f(n+1) \lambda^{n+1}<r \leq f(n) \lambda^{n}$. Since $\log f(n) / n \rightarrow 0$, we deduce

$$
\begin{aligned}
& \liminf _{r \rightarrow 0} \frac{\log \mu(B(x, r)}{\log r}=\liminf _{n \rightarrow \infty} \frac{\log \mu\left(B\left(x, f(n) \lambda^{n}\right)\right)}{\log f(n) \lambda^{n}} \\
= & \liminf _{n \rightarrow \infty} \frac{\log \mu\left(B\left(x, f(n) \lambda^{n}\right)\right)}{\log \lambda^{n}}=\liminf _{n \rightarrow \infty} \frac{\log P_{n}\left(\Delta_{n}(x)\right)}{n \log \lambda} .
\end{aligned}
$$

Thus $\underline{\operatorname{dim}}_{\mathrm{loc}} \mu(x)$ is as claimed.

The arguments for the upper local dimension are identical and left to the reader.

Example 5.13. A measure $\mu$ with $\operatorname{dim}_{\mathrm{qA}} \mu=\sup _{x}\left\{\overline{\operatorname{dim}}_{\text {loc }} \mu(x)\right\}$ that is not generalized regular: Consider the IFS $S_{0}(x)=x / 3, S_{1}(x)=x / 3+1 / 3, S_{2}(x)=x / 3+2 / 3$, with probabilities $p_{0}=p_{2}=2 / 5, p_{1}=1 / 5$ and associated self-similar measure $\mu$. This is a comparable, but not generalized regular, iterated function system. Note that $Q_{n}(x) \leq 5^{n}$ for all $x$ and $n$. Thus Theorem 5.12 yields that $\operatorname{dim}_{\mathrm{qA}} \mu \leq \log 5 / \log 3$, which coincides with $\operatorname{dim}_{\text {loc }} \mu(1 / 2)$, the maximum upper local dimension. Since the quasi-Assouad dimension is always an upper bound on the upper local dimension of the measure, we have equality here.

It would be desirable to know if all weakly comparable measures $\mu$ have the property that $\operatorname{dim}_{\mathrm{qA}} \mu=\sup \left\{\overline{\operatorname{dim}}_{\mathrm{loc}} \mu(x): x \in[0,1]\right\}$. 


\subsection{An equicontractive, regular, self-similar measure without full support.}

Throughout this paper, we have assumed that the self-similar measure has the full interval $[0,1]$ as its support. The purpose of the final example is to show that even an equicontractive, regular measure without full support need not have finite quasi-Assouad dimension: Consider the iterated function system with $S_{j}(x)=x / 5+d_{j}$ where $d_{0}=0, d_{1}=1 / 10, d_{2}=2 / 5$, $d_{3}=4 / 5$ and probabilities $p_{0}=p_{1}=p_{3}=1 / 6, p_{2}=1 / 2$. This IFS is equicontractive, finite type and regular, but the self-similar set is clearly not the full interval $[0,1]$. Indeed, the subintervals $(3 / 10,2 / 5)$ and $(3 / 5,4 / 5)$ are in the complement of the self-similar set.

As explained in [11, we can associate with each net interval of a finite type IFS a finite tuple called the characteristic vector. The characteristic vector contains all the information needed to essentially determine the measure of the net interval given that of its parent net interval. In fact, finite type is characterized by the property that there are only finitely many of these so-called characteristic vectors and, furthermore, each net interval $\Delta_{n}$ of level $n$ can be uniquely identified by the $(n+1)$-tuple of characteristic vectors, $\left(\gamma_{j}\right)_{j=0}^{n}$, where $\gamma_{n}$ is the characteristic vector of $\Delta_{n}, \gamma_{n-1}$ is the characteristic vector of its parent net interval, $\Delta_{n-1}$, etc.

This IFS has six characteristic vectors, which we label as $1,2,3 a, 3 b, 3 c, 4$. Any net interval of level $n-1$ with characteristic vector $3 a, 3 b$, or $3 c$ has four children, each of length $5^{-n} / 2$. From left to right these are $3 a, 3 b, 4,3 c$, where 4 and $3 c$ are separated by a gap. Fix $\varepsilon>0$ and take any large $N$ and $n=\lceil(1+\delta) N\rceil$. Consider the level $n$ net interval, $\Delta_{n}$, identified with the tuple,

$$
(1, \underbrace{3 a, 3 a, \ldots, 3 a}_{N}, \underbrace{3 b, 3 b, \ldots, 3 b}_{n-N})
$$

and its ancestor $\Delta_{N}$ of level $N$. Let $x_{n}$ denote the midpoint of $\Delta_{n}$.

Using the techniques of [11] it can be shown that

$$
\mu\left(\Delta_{N}\right) \sim\left\|[1 / 61 / 6]\left[\begin{array}{cc}
1 / 6 & 0 \\
0 & 1 / 2
\end{array}\right]^{N}\right\| \sim 2^{-N},
$$

while

$$
\mu\left(\Delta_{n}\right) \sim\left\|[1 / 61 / 6]\left[\begin{array}{cc}
1 / 6 & 0 \\
0 & 1 / 2
\end{array}\right]^{N}\left[\begin{array}{cc}
1 / 6 & 1 / 6 \\
0 & 0
\end{array}\right]^{n-N}\right\| \sim 6^{-n},
$$

where the symbol $\sim$ means bounded above and below by some constant multiple. Taking $R=5^{-N} / 2$ and $r=5^{-n} / 4$ it follows that

$$
\frac{\mu\left(B\left(x_{n}, R\right)\right)}{\mu\left(B\left(x_{n}, r\right)\right)} \geq c_{1} \frac{2^{-N}}{6^{-n}} \geq c_{1}^{\prime} \frac{6^{(1+\delta) N}}{2^{N}}=c_{1}^{\prime \prime} 3^{N} 6^{\delta N},
$$

while $R / r \leq c_{2} 5^{\delta N}$. Consequently, $\operatorname{dim}_{\mathrm{qA}} \mu=\infty$.

\section{REFERENCES}

[1] P. Assouad. Espaces métriques, plongements, facteurs, Thèse de doctorat d'État, Publ. Math. Orsay 223-7769, Univ. Paris XI, Orsay, (1977).

[2] D-J. Feng, Smoothness of the $L^{q}$-spectrum of self-similar measures with overlaps, J. London Math. Soc. 68 (2003), 102-118.

[3] D-J. Feng, The limited Rademacher functions and Bernoulli convolutions associated with Pisot numbers, Adv. in Math., 195 (2005), 24-101. 
[4] D-J. Feng, Multifractal analysis of Bernoulli convolutions associated with Salem numbers, Adv. Math. 229 (2012), 3052-3077.

[5] J. M. Fraser. Assouad type dimensions and homogeneity of fractals, Trans. Amer. Math. Soc. 366 (2014), 6687-6733.

[6] J. M. Fraser, A. M. Henderson, E. J. Olson, and J. C. Robinson, On the Assouad dimension of selfsimilar sets with overlaps, Adv. Math. 273 (2015), 188-214.

[7] J. M. Fraser and D. Howroyd, On the upper regularity dimension of measures, Indiana Univ. Math. J. (to appear), arXiv:1706.09340

[8] J. M. Fraser and S. Troscheit. The Assouad spectrum for random self-affine sets, Mittag-Leffler preprints 14801 (2018), arXiv:1805.04643

[9] I. García and K. E. Hare. Properties of Quasi-Assouad dimension, Camb. Proc. Phil. Soc. (to appear), arXiv:1703.02526

[10] K.E. Hare, K.G. Hare, K.R. Matthews, Local dimensions of measures of finite type, J. Fractal Geometry 3 (2016), 331-376.

[11] K.E. Hare, K.G. Hare and M. K-S. Ng, Local dimensions of measures of finite type II - measures without full support and with non-regular probabilities, Can. J. Math. 70 (2018), 824-867.

[12] K.E. Hare, K.G. Hare and G. Simms, Local dimensions of measures of finite type III - measures that are not equicontractive, J. Math. Anal. and Appl. 458 (2018), 1653-1677.

[13] A. Käenmäki and J. Lehrbäck, Measures with predetermined regularity and inhomogeneous self-similar sets, Ark. Mat. 55 (2017), 165-184.

[14] A. Käenmäki, J. Lehrbäck and M. Vuorinen, Dimensions, Whitney covers, and tubular neighborhoods, Indiana Univ. Math. J. 62 (2013), 1861-1889.

[15] K.-S. Lau and S.-M. Ngai, Multifractal measures and a weak separation condition, Adv. Math. 141 (1999), 45-96.

[16] J. Luukkainen. Assouad dimension: antifractal metrization, porous sets, and homogeneous measures, J. Korean Math. Soc. 35 (1998), 23-76.

[17] F. Lü and L. Xi. Quasi-Assouad dimension of fractals, J. Fractal Geom. 3 (2016), 187-215.

[18] S-M. Ngai and Y. Wang, Hausdorff dimension of self-similar sets with overlaps, J. London Math. Soc. 63 (2001), 655-672.

[19] J. C. Robinson. Dimensions, Embeddings, and Attractors, Cambridge University Press, (2011).

[20] Y. Peres, W. Schlag and B. Solomyak, Sixty years of Bernoulli convolutions, Fractal geometry and stochastics, II, Progress in probability 46, Birkhäuser, Basel, 2000, 39-65.

[21] P. Shmerkin, On the exceptional set for absolute continuity of Bernoulli convolutions, Geom. Func. Anal., 24 (2014), 946-958.

[22] P. Shmerkin, A modified multi-fractal formalism for a class of self-similar measures with overlap, Asian J. Math., 9 (2005), 323-348.

[23] M. P. W. Zerner. Weak separation properties for self-similar sets, Proc. Amer. Math. Soc. 124 (1996), 3529-3539.

Kathryn E. Hare, Department of Pure Mathematics, University of Waterloo, Canada. E-mail address: kehare@uwaterloo.ca

Kevin G. Hare, Department of Pure Mathematics, University of Waterloo, Canada.

E-mail address: kghare@uwaterloo.ca

Sascha Troscheit, Department of Pure Mathematics, University of Waterloo, Canada.

E-mail address: stroscheit@uwaterloo.ca 